Projets

de paysage

\section{Projets de paysage}

Revue scientifique sur la conception et l'aménagement de l'espace

$8 \mid 2012$

Les concepteurs de jardins et de parcs japonais

\title{
Kobori Enshū : deux biographies, une légende
}

Étude sur l'histoire des jardins japonais à travers l'œuvre de Shigemori Mirei et de Mori Osamu

Kobori Enshū : two biographies, one legend - A study on Japanese gardens

history through the works of Shigemori Mirei and Mori Osamu

\section{Emmanuel Marès}

\section{OpenEdition}

\section{Journals}

Édition électronique

URL : http://journals.openedition.org/paysage/14721

DOI : 10.4000/paysage. 14721

ISSN : 1969-6124

Éditeur :

École nationale supérieure du paysage de Versailles-Marseille, Institut national des sciences appliquées Centre Val de Loire - École de la nature et du paysage, École nationale supérieure d'architecture et de paysage de Bordeaux, École nationale supérieure d'architecture et de paysage de Lille, Agrocampus Angers

\section{Référence électronique}

Emmanuel Marès, « Kobori Enshū : deux biographies, une légende », Projets de paysage [En ligne], 8 2012, mis en ligne le 07 juillet 2012, consulté le 11 mars 2021. URL : http://journals.openedition.org/ paysage/14721 ; DOI : https://doi.org/10.4000/paysage.14721

Ce document a été généré automatiquement le 11 mars 2021.

Projets de paysage 


\title{
Kobori Enshū : deux biographies, une légende
}

\author{
Étude sur l'histoire des jardins japonais à travers l'œuvre de Shigemori \\ Mirei et de Mori Osamu \\ Kobori Enshū : two biographies, one legend - A study on Japanese gardens \\ history through the works of Shigemori Mirei and Mori Osamu
}

\section{Emmanuel Marès}

1 Dans cet article, il sera question de trois hommes, qui ont en commun le fait d'avoir été paysagistes. Parmi eux, nous porterons un intérêt tout particulier à la vie et à l'œuvre de Kobori Enshū 小堀遠州 (1579-1647), qui est sans aucun doute le paysagiste le plus célèbre de l'histoire des jardins japonais. Apprécié et respecté déjà de son vivant, le temps en a fait une légende. Il tient aujourd'hui une place de choix dans le panthéon des artistes japonais.

2 Mais en réalité, ce personnage historique et le manteau de mystère qui l'entoure sont avant tout un prétexte pour analyser et comparer les deux biographies que lui ont consacrées deux chercheurs et paysagistes du xx siècle: Shigemori Mirei 重森三玲 (1896-1975) et Mori Osamu 森蘊 (1905-1988). Le but de ce travail est donc de mieux comprendre le regard que portaient ces deux hommes sur Kobori Enshū et, à travers lui, sur l'histoire des jardins japonais en général.

\section{Kobori Enshū, un personnage hors du commun}

Kobori Sakusuke Masakazu, dit Kobori Enshū, était un seigneur (daimyō) au service de la dynastie militaire des Tokugawa au début du XVII ${ }^{\mathrm{e}}$ siècle. En plus de ses fonctions administratives officielles, il a su déployer son talent d'architecte, de concepteur de jardins, de maître de thé, de poète, de calligraphe... C'était un artiste qui excellait dans tous les domaines.

4 À la fin de sa vie, il fut nommé maître de thé personnel du $3^{\mathrm{e}}$ shogun Tokugawa Iemitsu 徳川家光 (1604-1651), une récompense qui montre l'aura que pouvait avoir Enshū dans 
les hautes sphères de la société de l'époque. Mais cela prouve surtout que malgré sa fonction principale de maître d'œuvre des constructions officielles (sakuji bugyō 作事奉 行), qui laissait peu de place à l'innovation et à l'originalité, il avait réussi le tour de force d'imposer une esthétique qui lui était propre. Il a su trouver un équilibre entre un style avant-gardiste et une esthétique respectueuse de la sensibilité de ses contemporains. En effet, alors que ses prédécesseurs, Sen no Rikyū 千利休 (1522-1591) et Furuta Oribe 古田織部 (1544-1615), se sont donné la mort sur ordre de leur seigneur, Kobori Enshū est le seul à avoir goûté à une fin de vie paisible.

Il a marqué son temps et son influence sur l'évolution des arts japonais jusqu'à aujourd'hui est considérable. Ses descendants ont formé «l'école Enshū » (Enshū rȳu sadō 遠州流茶道) et ils continuent aujourd'hui de perpétuer l'art de la cérémonie du thé tel que leur ancêtre l'a élaboré. Le seul nom d'«Enshū» continue d'attirer les foules, comme le montre le succès de l'exposition qui lui a été consacrée de fin 2007 à début 2008 et qui a rassemblé plus de 140000 visiteurs $^{1}$. De même, tous les jardins attribués à Enshū à travers le Japon connaissent toujours une grande affluence. Plus de trois cent cinquante ans après sa disparition, son art est encore d'actualité et fait toujours l'unanimité.

Depuis, de nombreux travaux ont été consacrés à cette période de l'histoire et à ce personnage hors du commun. Mais parmi toutes ces publications, deux biographies font référence :

- Shigemori Mirei 重森三玲, Kobori Enshū 小堀遠州, Kyōto, Kawara shoten 河原書店, Chajinden sōsho 2 茶人伝盖書〈第2〉(Collection biographique des maîtres de thé), 1949, $274 \mathrm{p}$. 
Figure 1. Couverture de la biographie de Kobori Enshū écrite par Shigemori Mirei en 1949

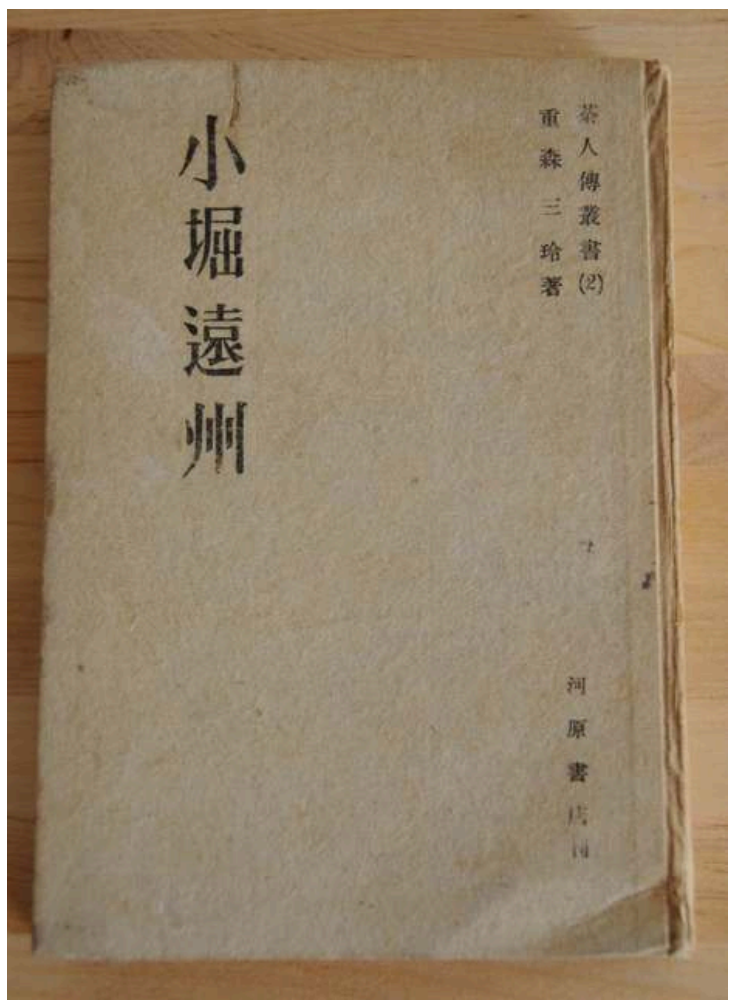

- Mori Osamu 森緧, Kobori Enshū 小堀遠州, Tōkyō, Yoshikawa kōbunkan 吉川弘文館, Jinbutsu sōsho 人物叢書 (Collection des grands personnages), 1967, 377 p.

Figure 2. Couverture de la première édition de la biographie de Kobori Enshū écrite par Mori Osamu en 1967

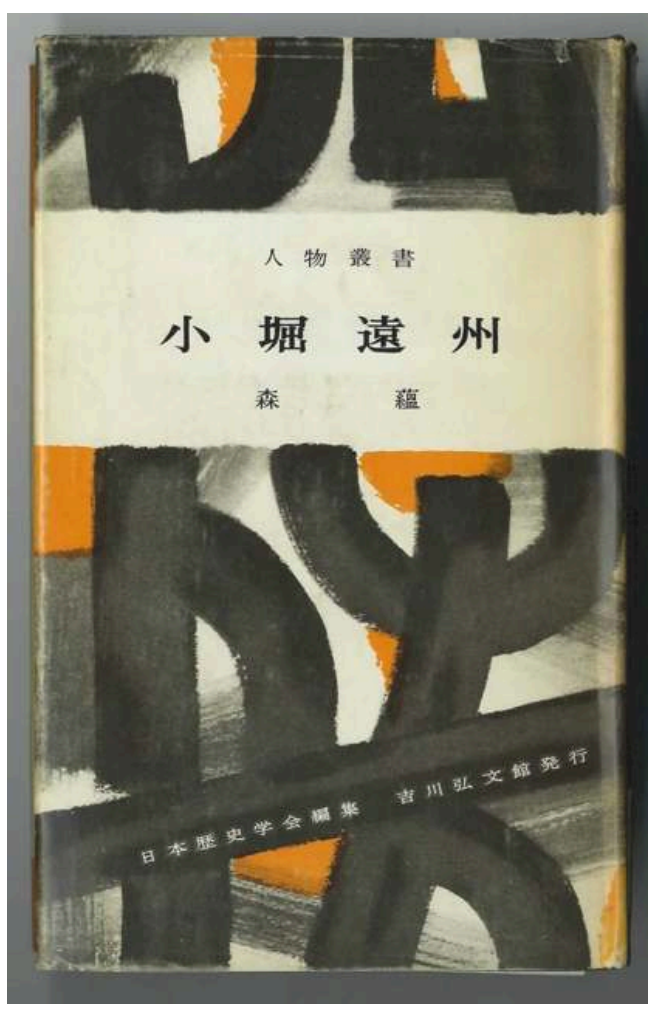


Figure 3. Couverture de la deuxième édition de la biographie de Kobori Enshū écrite par Mori Osamu (2003)

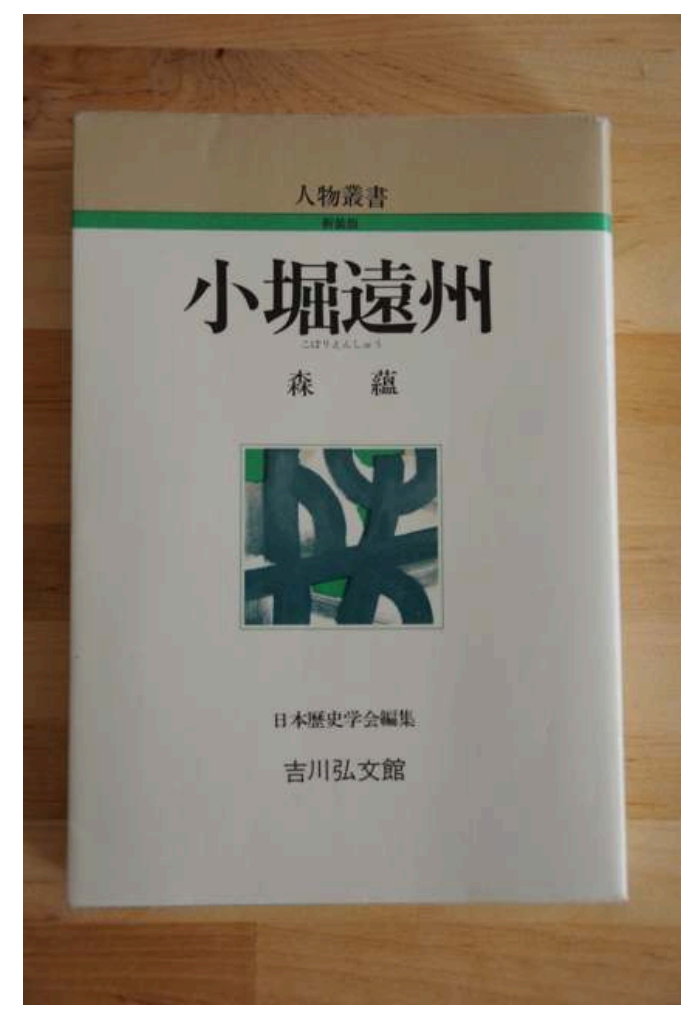

Daimyō, maître d'œuvre des constructions officielles, grand maître de thé et artiste aux multiples facettes, Kobori Enshū aurait dû susciter l'intérêt de spécialistes de l'histoire de l'art, de l'architecture ou encore de la cérémonie du thé. Et pourtant, ces deux livres ont été écrits par deux spécialistes du jardin. À presque vingt ans d'intervalle et chez deux éditeurs différents, il est assez étonnant que ce soit des paysagistes et des historiens des jardins qui aient choisi de prendre cette lourde responsabilité : écrire la biographie de Kobori Enshū.

Pour comprendre l'histoire des jardins japonais, est-il possible de faire l'impasse sur la vie et l'œuvre de ce personnage? Au Japon, rares sont les jardiniers-paysagistes qui laissent leur nom à la postérité. Kobori Enshū fait partie de ces quelques figures qui viennent marquer l'histoire des jardins. En plus des vestiges relativement bien conservés, il existe de nombreux écrits qui témoignent du caractère monumental de son travail. De son vivant déjà, il était décrit comme une icône incontournable.

À ce propos, il est intéressant de noter qu'il s'agit de la seule biographie publiée par les deux auteurs. Par la suite, ils publieront de nombreux livres sur l'art et l'histoire des jardins, mais rien à propos d'un autre personnage historique. Une preuve supplémentaire de l'aura que pouvait avoir Kobori Enshū dans ce domaine.

10 À première vue, on pourrait imaginer que ces deux livres se ressemblent puisque les auteurs, en plus d'être des contemporains, sont des historiens de l'art des jardins japonais. Il n'en est rien. En réalité, ce sont deux hommes que tout oppose. Shigemori Mirei était un autodidacte, un artiste visionnaire et inspiré, alors que Mori Osamu était un universitaire de formation, historien et archéologue méticuleux. De leur vivant déjà, 
la concurrence était vive et lorsque l'un affirmait une chose, l'autre cherchait à prouver le contraire.

11 Avec le recul, il nous est permis aujourd'hui d'aller au-delà de cette rivalité et de faire la synthèse de leurs travaux pour mieux comprendre le passé, mais aussi pour ouvrir de nouvelles perspectives à la recherche dans ce domaine. Avant tout, il semble important d'esquisser le parcours de ces deux hommes, une première étape qui nous permettra de mieux appréhender leur point de vue respectif.

\section{Shigemori Mirei et Mori Osamu, l'artiste et le savant}

12 Alors que de son temps Shigemori Mirei a longtemps travaillé dans l'indifférence de ses contemporains, il est devenu aujourd'hui le paysagiste d'après-guerre le plus connu du grand public. Depuis quelques années, son travail fait l'objet d'un regain d'intérêt et l'une de ses créations, le jardin de M. Nishida (Seiryōtei 青龍庭 le jardin du Dragon bleu) dans le département d'Osaka, a été désigné comme propriété culturelle du Japon en 2008. C'est, à n'en pas douter, le premier d'une longue liste qui va aller en s'étoffant dans les années à venir.

Quelques dates qui ont marqué sa vie: En 1911, Shigemori Mirei commence l'apprentissage de la cérémonie du thé et de l'ikebana, il a quatorze ans. À peine quatre ans plus tard, il dessine son premier pavillon de thé (Tenrai-an 天藾庵). C'est son père, charpentier à ses heures perdues, qui le construira à côté de leur maison ${ }^{2}$. Il démontre ainsi deux choses : tout d'abord, sa capacité à intégrer un grand nombre d'informations en très peu de temps. De plus, il illustre un principe qui deviendra par la suite une certitude : la tradition n'est pas quelque chose de figé dans le temps mais une source d'inspiration pour créer des concepts avant-gardistes. Pour devenir un classique, il faut d'abord être un révolutionnaire.

En 1934, Mirei a trente-huit ans et s'est installé à Kyōto. Lorsque le typhon Muroto dévaste la région du Kansai, il réalise qu'il n'existe aucun plan original pour réaménager les jardins et sites célèbres en cas de catastrophe. Il entreprend donc de faire le relevé de tous les jardins historiques du Japon. De 1936 à 1939, il parcourt le Japon du nord au sud et recense environ trois cent cinquante jardins historiques. Il publiera le résultat de ce travail titanesque dans un ouvrage en vingt-six volumes intitulé : Nippon teienshi zukan 日本庭園史困鑑 (L'Histoire illustrée des jardins japonais). C'est la première histoire complète des jardins japonais ${ }^{3}$.

En 1939, alors qu'il est en train de faire les derniers relevés dans le temple Tōfuku-ji, le supérieur lui demande de concevoir un jardin autour du pavillon principal. Pour un premier travail commandité, c'est un chantier de grande envergure, le Tōfuku-ji étant un des principaux temples Zen de Kyōto. Mirei ne se laisse pas effrayer par l'enjeu et il laisse libre cours son imagination. Il crée quatre jardins secs alliant tradition et design avant-gardiste tout autour du pavillon principal. C'est un premier succès, et cette œuvre est devenue aujourd'hui une référence incontournable du jardin japonais moderne. 
Figure 4. Jardin sud du pavillon principal du temple Tōfuku-ji à Kyoto

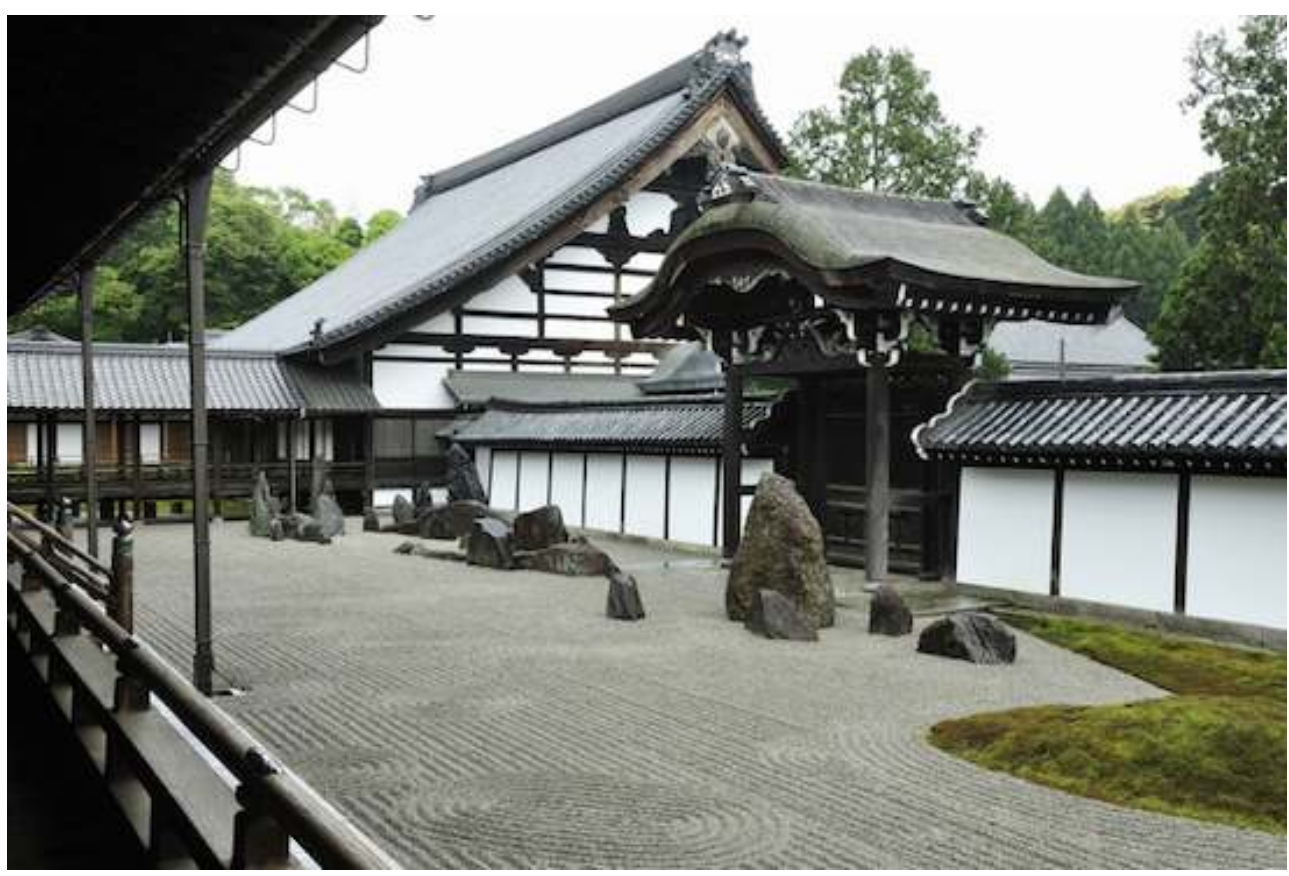

Chaque groupe de pierres représente une île mythique, où vivent les immortels chinois : Hōrai, Hōjō, Eishū, Koryō (la quatrième île est hors cadre et ne figure donc pas sur la photo).

Source : Temple Tōfuku-ji.

Figure 5. Jardin ouest du pavillon principal du temple Tōfuku-ji à Kyoto

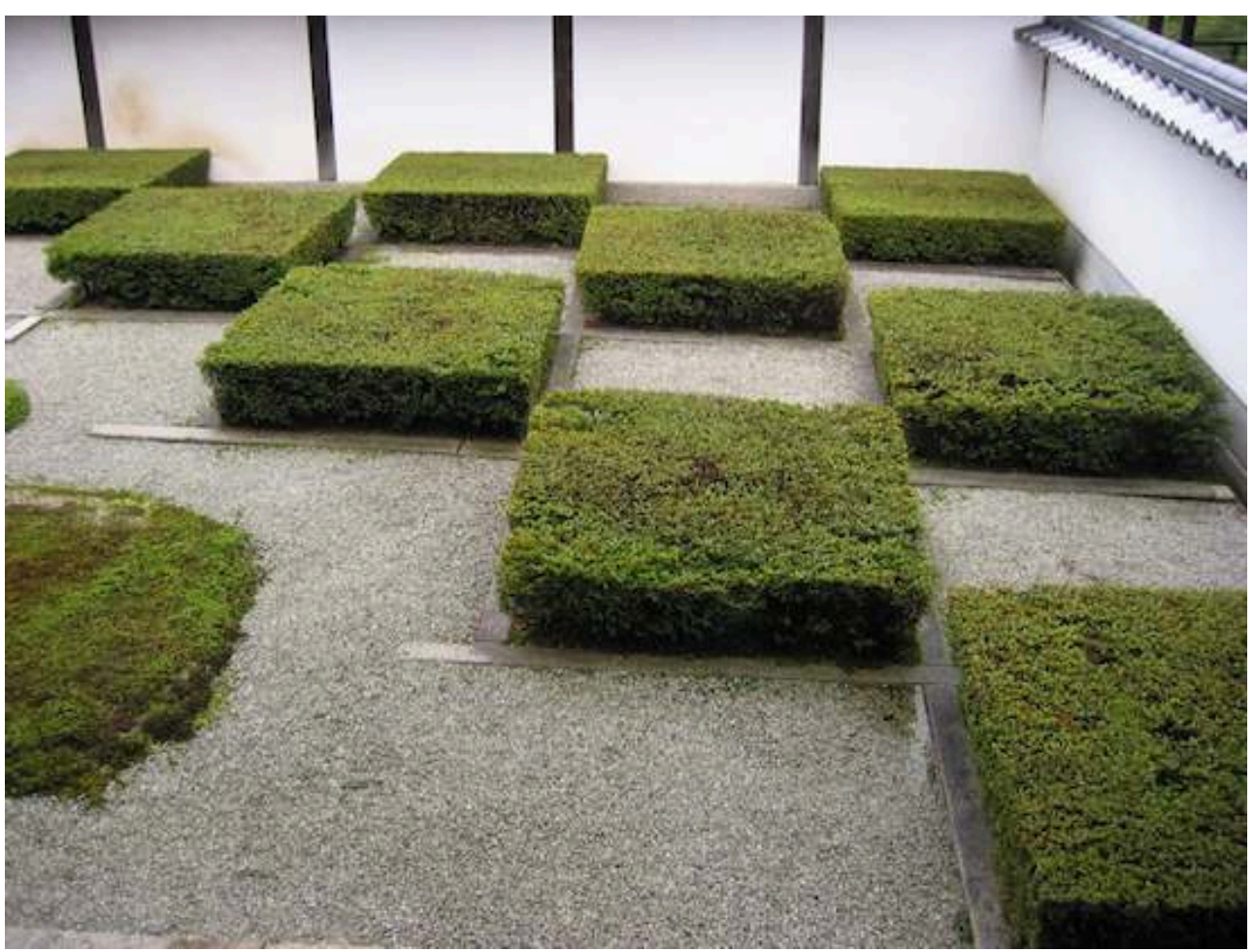

Damier en trois dimensions composé de massifs d'azalées taillées et de carrés de gravier blanc délimités par des pierres taillées.

Source :Temple Tōfuku-ji. 
Figure 6. Jardin nord du pavillon principal du temple Tōfuku-ji à Kyoto

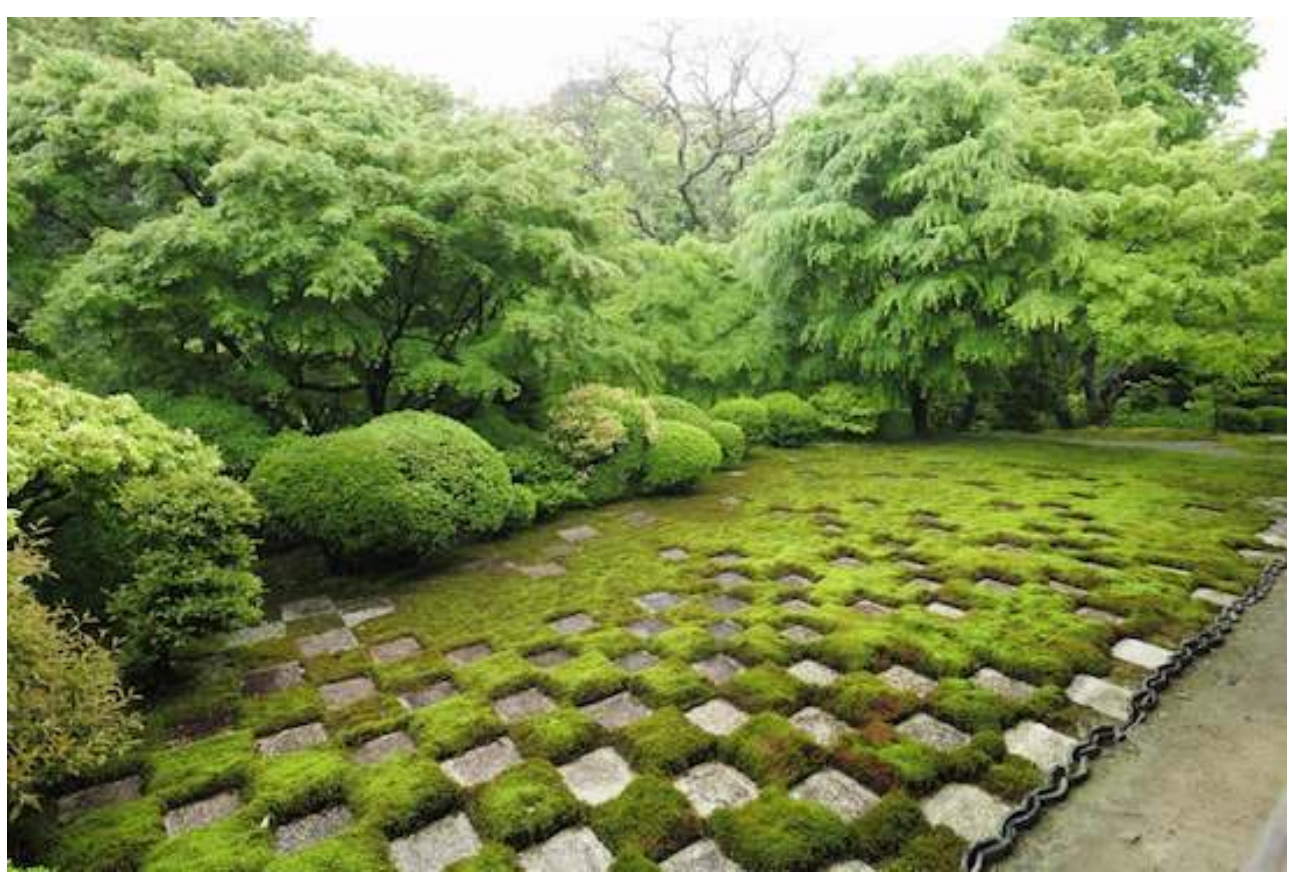

Shigemori Mirei a réutilisé des dalles qui se trouvaient dans le temple pour composer un damier avec des pierres et de la mousse.

Source : Temple Tōfuku-ji.

Mirei réduit le végétal à sa plus simple expression, il n'hésite pas à utiliser des formes géométriques et joue sur le contraste entre horizontalité et verticalité. Pour chacun de ces jardins, il définit un thème et une symbolique très forte. Ce projet a scellé son succès et son destin. Il pose sa plume et, jusqu'à sa mort en 1975, il va se consacrer principalement à la création de jardins.

17 Sans aucun lien avec une quelconque école ou lignée, Shigemori Mirei a su revisiter la tradition à sa façon. Ce n'était pas un artisan-jardinier mais un artiste, pour qui le jardin est avant tout une œuvre d'art ${ }^{4}$, d'où l'importance de faire des relevés pour retrouver la forme "originale" des jardins historiques. Le jardin était pour lui un espace de recherche purement esthétique, une feuille blanche (tabula rasa), sur laquelle l'artiste devait apposer sa signature. Le travail de toute sa vie a donc été de redonner ses lettres de noblesse au jardin japonais traditionnel qui, dans le prisme de la conception de l'art occidental, était devenu un art mineur par rapport à l'architecture.

Mori Osamu n'a pas eu un parcours aussi théâtral. Scientifique de formation, il a consacré sa vie aux jardins et, effectivement, toutes ses publications portent sur l'art et l'histoire des jardins japonais ${ }^{5}$. Aujourd'hui, ses travaux font référence dans ce domaine, mais le personnage est totalement inconnu du grand public et son travail en tant que paysagiste est tombé dans l'oubli.

Quelques moments clés de sa vie: Mori Osamu s'inscrit à la faculté d'agronomie de l'université impériale de Tōkyō en 1929. Il va suivre principalement les cours sur l'histoire de l'art, de l'architecture et du paysagisme. Parmi tous ses professeurs, il va suivre tout particulièrement Tamura Tsuyoshi 田村剛 (1890-1979), spécialiste des jardins et des parcs nationaux. Simple coïncidence ou signe du destin? Il s'agit du 
même Tamura Tsuyoshi qui a écrit quelques années plus tôt un article qui ne tarit pas d'éloges sur le tout premier jardin conçu par Shigemori Mirei ${ }^{6}$.

À la mort de son père en 1933, Mori Osamu quitte l'université. Parallèlement à son nouveau travail de fonctionnaire, il poursuit les recherches qu'il avait entamées sur les jardins de l'époque de Heian (794-1185) et publie le résultat de son travail en $1945^{7}$. En 1952, il devient directeur de recherche à l'Institut national de recherche de Nara pour les propriétés culturelles importantes. Deux ans plus tard, il présente sa thèse sur l'histoire du jardin de la villa Katsura et obtient son doctorat, il a 47 ans.

21 Dans son travail, il reprend la thèse de Sotoyama Eisaku 外山英策 (1888-1953) $)^{8}$, qui s'appuyait sur l'analyse de documents historiques pour prendre le contre-pied d'une assertion qui faisait alors l'unanimité, à savoir que Kobori Enshū aurait été le concepteur des bâtiments et du jardin de la villa Katsura. Mori Osamu achève de démonter ce mythe en élargissant ses recherches : il organise des fouilles et établit des relevés précis des bâtiments et du jardin. Tout son travail en tant que chercheur s'inscrit dans cette perspective : à partir de l'analyse des documents d'époque et du résultat des recherches archéologiques, il cherche à percer les secrets de l'histoire des jardins japonais.

Mais dans l'ombre de cette œuvre académique se cache sa deuxième vocation: Mori Osamu était aussi paysagiste. Il a travaillé pour des temples prestigieux à Nara mais ses jardins sont rapidement tombés dans l'oubli. Ils n'ont peut-être pas la puissance évocatrice des créations de Shigemori Mirei qui connaissent un renouveau aujourd'hui. Quoi qu'il en soit, pour comprendre le résultat des recherches de Mori Osamu, il nous faudra, à un moment ou à un autre de cette étude, faire la lumière sur ce travail méconnu. Mais dans le cadre de cet article, nous nous attacherons seulement à l'analyse des deux biographies de Kobori Enshū. 
Figure 7. Jardin privé de la famille Iwai

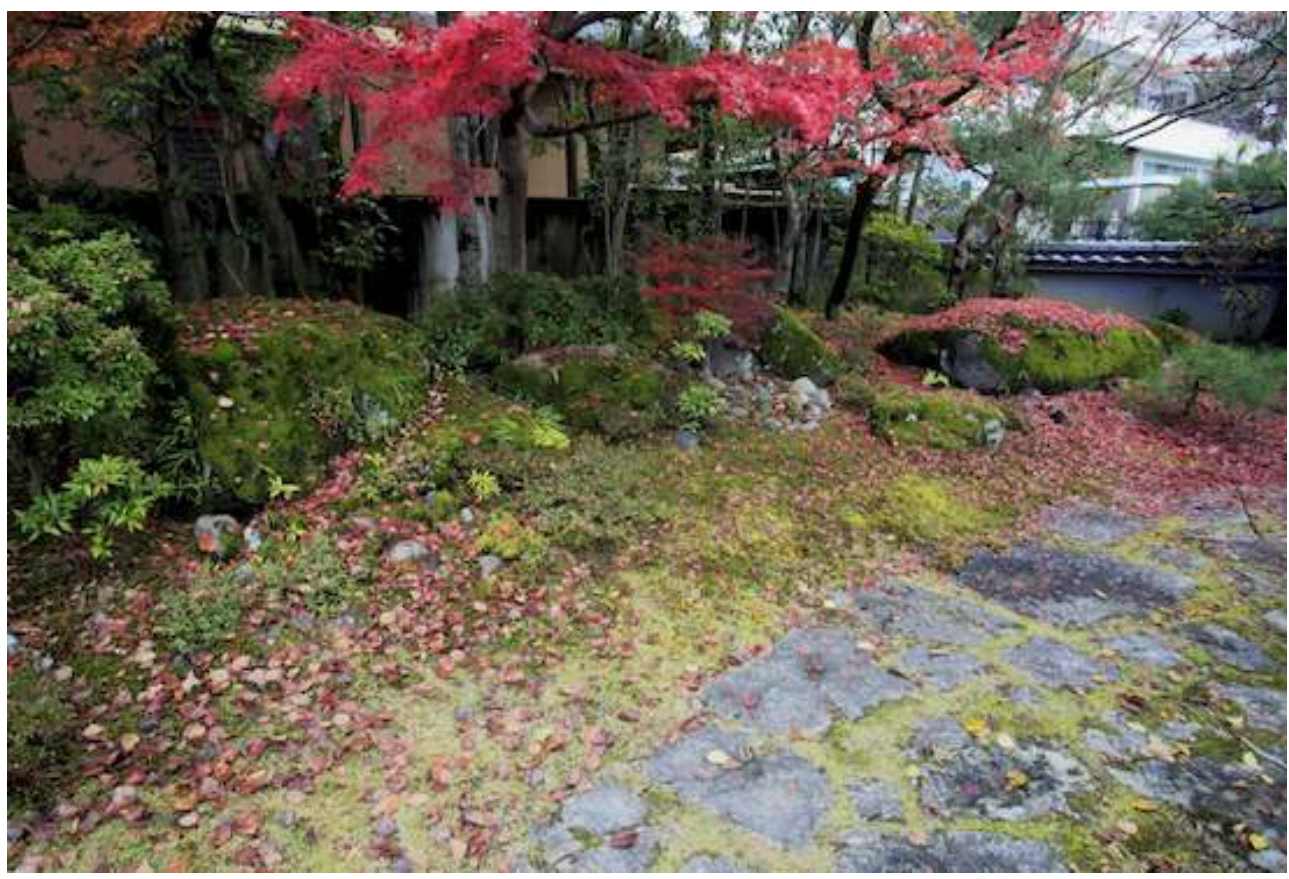

Mori Osamu a enterré le plus possible les immenses pierres qui étaient déjà dans le jardin pour adoucir l'ambiance de cet espace de vie. Aujourd'hui, la mousse a pratiquement recouvert toute la composition de pierres et crée une atmosphère apaisée.

Source : Fujii Kinji. 
Figure 8. Jardin privé de la famille Fukuyama

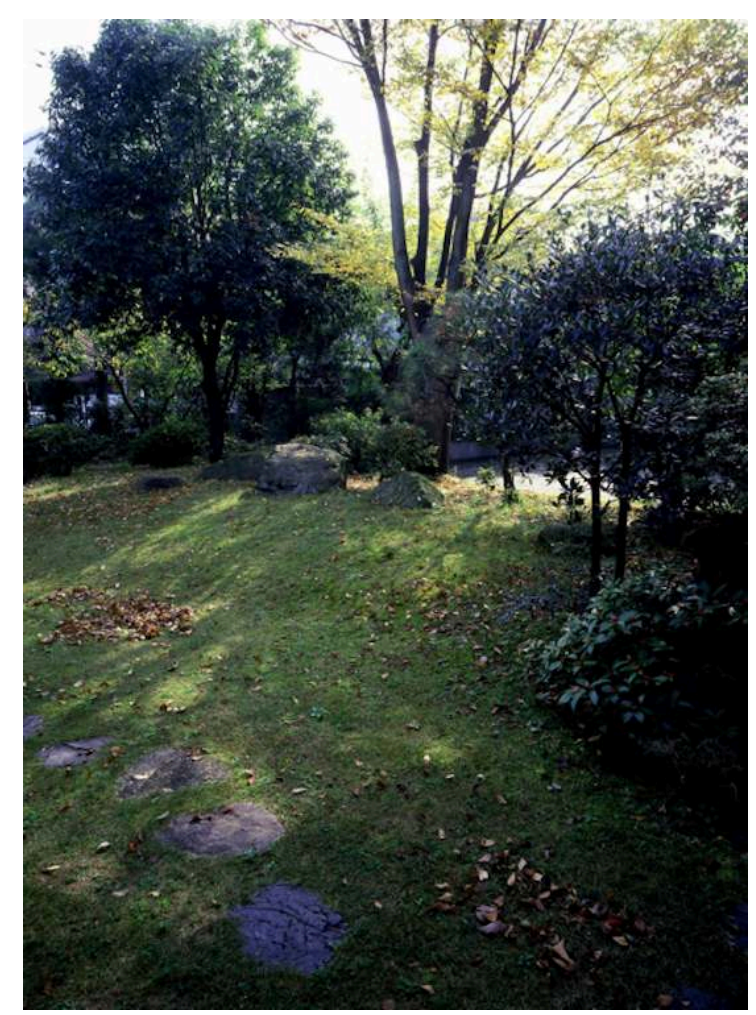

Mori Osamu apprécie les compositions simples : une allée en pierre et un arrangement de pierres qui a plutôt tendance à s'effacer dans la structure d'ensemble. II n'y a aucune pierre dressée.

Source : Fujii Kinji.

\section{Deux poids, deux mesures}

Kobori Enshū de Shigemori Mirei est paru aux éditions Kawara shoten, à Kyōto, en 1949. Il s'agit du deuxième numéro de la "Collection biographique des maîtres de thé » qui avait commencé un an plus tôt, en 1948. Le troisième volume ne verra cependant pas le jour, l'éditeur, rencontrant des problèmes d'approvisionnement en papier, aurait dû renoncer à son projet ${ }^{9}$. Il faut rappeler que le Japon sortait tout juste d'une guerre qu'il avait perdue et qui lui avait coûté très cher, économiquement, politiquement et humainement. Ce n'était pas une période faste pour l'édition et d'ailleurs le livre de Mirei est de facture très simple.

Ce livre n'a jamais été réédité. Il est difficile d'expliquer ce phénomène mais le style d'écriture obsolète est peut-être un élément de réponse. Il faut savoir qu'en 1946, le gouvernement a annoncé officiellement l'adoption des 1850 caractères chinois usuels simplifiés et un nouvel usage du syllabaire japonais. L'objectif de cette réforme était de simplifier la langue écrite mais de nombreux chercheurs, écrivains et autres lettrés se sont exprimés contre cette évolution. À l'origine, cette loi ne s'appliquait qu'aux manuels scolaires ou autres documents officiels et l'habitude d'écrire avec l'ancienne graphie a perduré quelques années. Le livre de Shigemori Mirei écrit en 1949 est donc le témoin de cette tendance mais ce choix le rend très difficile d'accès aujourd'hui.

Il semblerait que Shigemori Mirei en personne se soit proposé pour écrire cette biographie. En 1949, les spécialistes de Kobori Enshū étaient Horiguchi Sutemi 堀口捨 
巳 (1895-1984) et Sawashima Eitarō 澤島英太郎 ${ }^{10}$. Shigemori Mirei n'a jamais été très actif dans le monde académique (il n'a jamais publié dans une revue scientifique), mais il avait déjà publié de nombreux livres sur la cérémonie du thé et l'ikebana. En outre, il ne faut pas oublier qu'il venait de terminer sa monumentale histoire des jardins japonais. Après toutes ces années passées à faire des relevés et à travailler sur les jardins, Mirei devait avoir son mot à dire sur un personnage aussi emblématique que Kobori Enshū. Écrire ce livre était peut-être pour lui une façon de répondre aux universitaires avec qui il était en désaccord, tout particulièrement à propos des nouvelles thèses qui remettaient en cause le lien de parenté entre Kobori Enshū et la villa Katsura.

Kobori Enshū de Mori Osamu est paru en 1967. Quelques années auparavant, en 1964, les premiers Jeux olympiques d'Asie étaient organisés à Tōkyō. Pour le Japon et les Japonais, c'est une date charnière. Ils ont enfin tourné la page de l'occupation américaine, de la reconstruction et ils reviennent sur l'échiquier international comme une puissance de premier plan.

Le livre de Mori Osamu a connu un destin très différent de celui de Shigemori Mirei : depuis sa date de parution en 1967, il a été réimprimé cinq fois. Bien évidemment, ce livre a été rédigé dès le départ en japonais moderne, ce qui peut expliquer qu'il soit toujours d'actualité, au moins par rapport au livre de Shigemori Mirei. La dernière impression date de 2003.

Cette biographie est le résultat d'une démarche spontanée. C'est le point final du travail qu'il avait commencé sur la villa Katsura en $1952^{11}$, car, comme il le souligne lui-même dans la préface, on ne peut pas essayer de comprendre le travail de Kobori Enshū en tant qu'architecte et en tant que paysagiste, sans appréhender l'homme dans son ensemble, en prenant compte de toutes ses différentes facettes. Le livre de Mori Osamu est donc né d'une nécessité d'aller plus loin et de faire la lumière sur la part de réalité et la part de mythe qui entouraient ce personnage hors du commun.

\section{Les jardins de Kobori Enshū, un thème de discorde}

Pour mieux comprendre le propos de chaque auteur, nous allons commencer par nous pencher sur la table des matières des deux livres (voir annexes I et II). Une première lecture nous permet déjà de distinguer deux attitudes radicalement opposées: Shigemori Mirei organise son livre autour de quatre grands thèmes alors que Mori Osamu suit un axe chronologique qu'il développe sur cinq chapitres.

Ce choix reflète bien le positionnement des deux auteurs, le premier est un artiste qui cherche à donner une image d'ensemble, ce qui a le mérite de rendre la lecture claire et accessible; le second est un scientifique qui veut donner des informations précises sur chaque moment de la vie de Kobori Enshū. Le défaut de cette seconde organisation, c'est peut-être le manque d'unité. Les informations sont distillées tout au long du livre et cela demande beaucoup d'efforts de la part du lecteur pour faire un travail de synthèse.

31 Sur 274 pages et quatre chapitres, Shigemori Mirei consacre 101 pages, soit plus d'un tiers, au dernier : "Enshū en tant que maître de thé ». Ce choix peut sembler évident pour un livre qui s'inscrit dans le cadre de la « Collection biographique des maitres de thé ». Ce qui est intéressant, c'est que malgré cet antécédent, Mori Osamu va lui aussi 
souligner que l'objectif principal de son livre est de faire le portrait de Kobori Enshū en tant que maitre de thé ${ }^{12}$. Mais cette prise de position est compréhensible si l'on se souvient qu'il avait publié à peine un an auparavant un livre exhaustif sur l'œuvre de Kobori Enshū : Kobori Enshū no sakuji (L'CEuvre de Kobori Enshū $)^{13}$.

Dans le cadre de cet article, nous ne pourrons pas discuter de toutes les divergences entre ces deux biographies. Malgré tout, il semble que les listes des jardins réalisés par Enshū soient assez révélatrices de ce qui peut opposer nos deux auteurs.

Dès le début de la section "Enshū paysagiste» (p.73-74) dans le second chapitre, Shigemori Mirei présente un inventaire précis :

• 1596, 19 ans, jardin de thé (roji) dans la résidence familiale d'Enshū, Rokujizō ;

• 1615, 37 ans, jardins du temple Raikyū-ji et Konekoya sho.in (conservé) ;

- 1627, 49 ans, jardin de thé (roji) du temple Konchi-in ;

- 1629, 51 ans, jardin de thé dans l'enceinte est du château d'Edo ;

-1630, 52 ans, jardin du temple Konchi-in (conservé) ;

- 1633, 55 ans, jardin du palais de l'empereur retiré Sentō gosho (conservé);

-1640, 62 ans, jardin du petit palais impérial Kogosho (conservé) ;

-1641, 63 ans, jardin de la villa Katsura (conservé) ;

-1643, 65 ans, jardin du temple Tōkaiji (conservé);

• 1643, 65 ans, (conservé) jardin du temple Kohō-an (conservé).

Mori Osamu, lui, ne donne pas une liste détaillée. Il classe toutes les réalisations de Kobori Enshū dans différentes catégories suivant la chronologie. Il est vrai qu'il est difficile de différencier totalement le travail d'Enshū en tant que paysagiste et en tant qu'architecte, les deux étant intimement liés. Dans une partie consacrée aux « vestiges des jardins attribués à Enshū » (p. 156-166), il liste cinq jardins qui se trouvent tous à Kyōto :

- le jardin du palais de l'empereur retiré, Sentō gosho ;

- le jardin de la deuxième enceinte du château de Nijō ;

- le jardin de la grue et de la tortue du temple Konchi-in ;

- le jardin sud du pavillon principal (hōjō) de Nanzen-ji ;

- le jardin du temple Kohō-an.

Plus loin, il reprend cette liste dans une partie intitulée «Les projets paysagers d'Enshū» (p. 193-203) qu'il classe selon cinq catégories: les jardins esthétiques, les jardins naturels et l'emprunt de paysage, les jardins pour les venues officielles, le jardin de thé (roji) et enfin, la réutilisation des pierres à l'intérieur du jardin de thé (roji). À l'intérieur de ce classement, Mori Osamu s'appuie sur des exemples concrets, que ce soit des vestiges de jardins ou bien des plans qu'il a retrouvés, pour essayer de montrer en quoi Kobori Enshū a bouleversé l'art du jardin japonais à son époque. Voici la liste des jardins qui ont aujourd'hui disparu :

- le petit palais impérial Kogosho ;

- le nouveau palais impérial Shin.in gosho ;

- le jardin de thé de la deuxième enceinte du château d'Edo ;

- le jardin de thé (roji) de la résidence familiale d'Enshū Rokujizō.

Avant toute chose, les auteurs ne sont pas d'accord sur le nombre de réalisations : 10 pour Shigemori Mirei et 9 pour Mori Osamu. Nous allons essayer de voir quels sont les points de discorde mais il est déjà intéressant de noter que pour un personnage qui est considéré comme le plus grand paysagiste dans l'histoire des jardins japonais, cela fait 
peu de réalisations. À titre de comparaison, on peut compter environ 65 jardins créés par Mori Osamu et presque 200 par Shigemori Mirei. Mais ce nombre est aussi révélateur du caractère exceptionnel de ce personnage, capable en seulement quelques créations de révolutionner un art déjà multiséculaire. Nous allons maintenant nous concentrer sur les différences que présentent ces deux listes. D'un côté, Mori Osamu ne cite pas les jardins du temple Raikyū-ji et Konekoya sho.in (1), ni celui de la villa Katsura (5). De l'autre, Shigemori Mirei ne liste pas les jardins du château de Nijō (2), du nouveau palais impérial Shin.in gosho (4), et ni celui du pavillon principal du temple Nanzen-ji (3). Sur quoi se fondent ces divergences?

(1) Les jardins du temple Raikyū-ji et Konekoya sho.in se situent dans la région d'Okayama, non loin de l'ancien château de Bichū Matsuyama, où le père d'Enshū a été nommé administrateur local en 1600. À la mort de son père en 1604, Enshū hérite de ce poste qu'il va occuper pendant plus d'une dizaine d'années.

Figure 9. Le jardin du temple Raikyū-ji

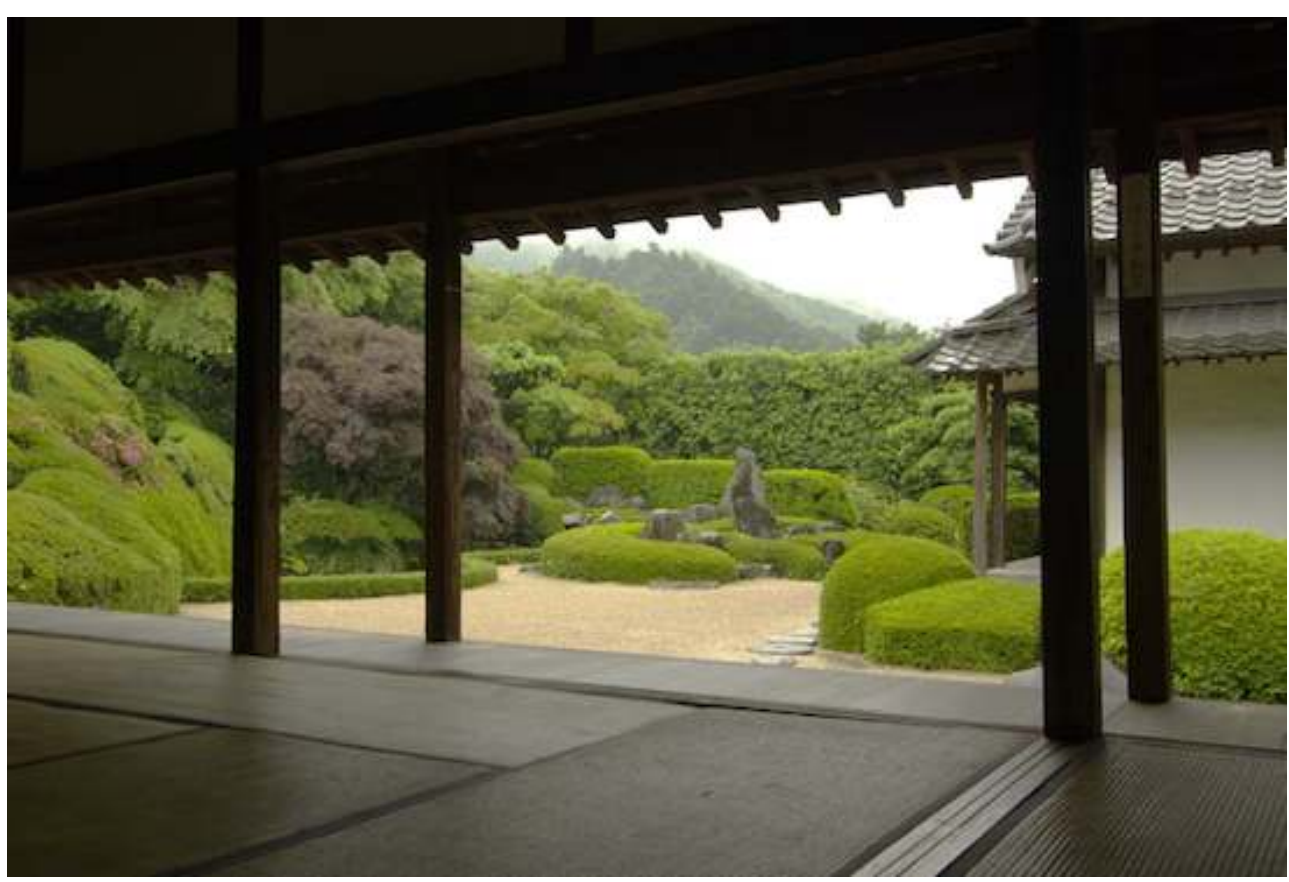

À l'origine, le thème de cette composition était la grue et la tortue. La pierre qui se dresse au milieu du jardin représente la grue. Malheureusement, l'arrangement de pierres représentant la tortue a disparu. Au fond, la haie est taillée de façon à évoquer le mouvement des vagues dans la mer. Il est difficile de savoir si cette haie était travaillée de cette façon du vivant de Kobori Enshū, au début du xvıe siècle.

Source : Kitaoka Shinya.

Le château était alors en très mauvais état et pendant les travaux de rénovation, il semblerait qu'Enshū ait élu domicile dans le temple Raikyū-ji et au Konekoya sho.in, un bâtiment qui a disparu aujourd'hui mais dont le jardin a été partiellement conservé à l'intérieur du lycée préfectoral de Takahashi. D’après les archives du temple Raikyū-ji, ce serait lors de son séjour dans ces résidences, entre 1614 et 1615, que Kobori Enshū aurait conçu ces deux jardins.

Pour Shigemori Mirei, cette hypothèse ne fait pas l'ombre d'un doute et il est intéressant de noter qu'elle est profondément ancrée dans l'imaginaire, comme on peut le constater en visitant les sites Internet du temple Raikyū-ji ou de Wikipédia qui 
attribuent les travaux de ces deux sites à Kobori Enshū. De plus, le descendant de Kobori Enshū, Kobori Sōkei le 12 du nom (1923-2011), a publié en 2010 un livre avec une photo du jardin de Raikyū-ji en couverture ${ }^{14}$. Il semblerait donc que cette association, Raikyū-ji = Enshū, ait encore l'assentiment du public et de certains experts.

Pourtant, dans le livre de Mori Osamu, ces deux jardins n'ont même pas le droit de cité. Bien évidemment, Mori Osamu prend le temps de décrire la période pendant laquelle Kobori Enshū a travaillé en tant qu'administrateur du château de Bichū Matsuyama mais l'hypothèse qu'il aurait pu concevoir les jardins du temple Raikyū-ji et Konekoya sho.in n'est même pas évoquée.

Dans son livre L'CEuvre de Kobori Enshū, il consacre un chapitre aux jardins attribués à Enshū et fait alors allusion à ces deux cas. Selon lui, Kobori Enshū devait être déjà suffisamment occupé avec les travaux du château et les troubles politiques liés au siège d'Osaka, où se sont tenues deux batailles entre 1614 et 1615, auxquelles Enshū a participé. Enfin, les archives du temple que cite Shigemori Mirei ont été écrites a posteriori et Mori Osamu ne les considère pas comme un témoignage fiable. Pour lui, il s'agit d'un raccourci trop facile.

43 (2) Les travaux du château de Nijō commencent en 1601. Lors des premiers travaux, un étang est creusé et un jardin construit juste devant le palais Ninomaru goten. À l'époque, Kobori Enshū n'est pas impliqué dans ce projet : il n'a que vingt-deux ans et n'a pas encore fait ses preuves.

Figure 10. Étang du château de Nijō vu du sud

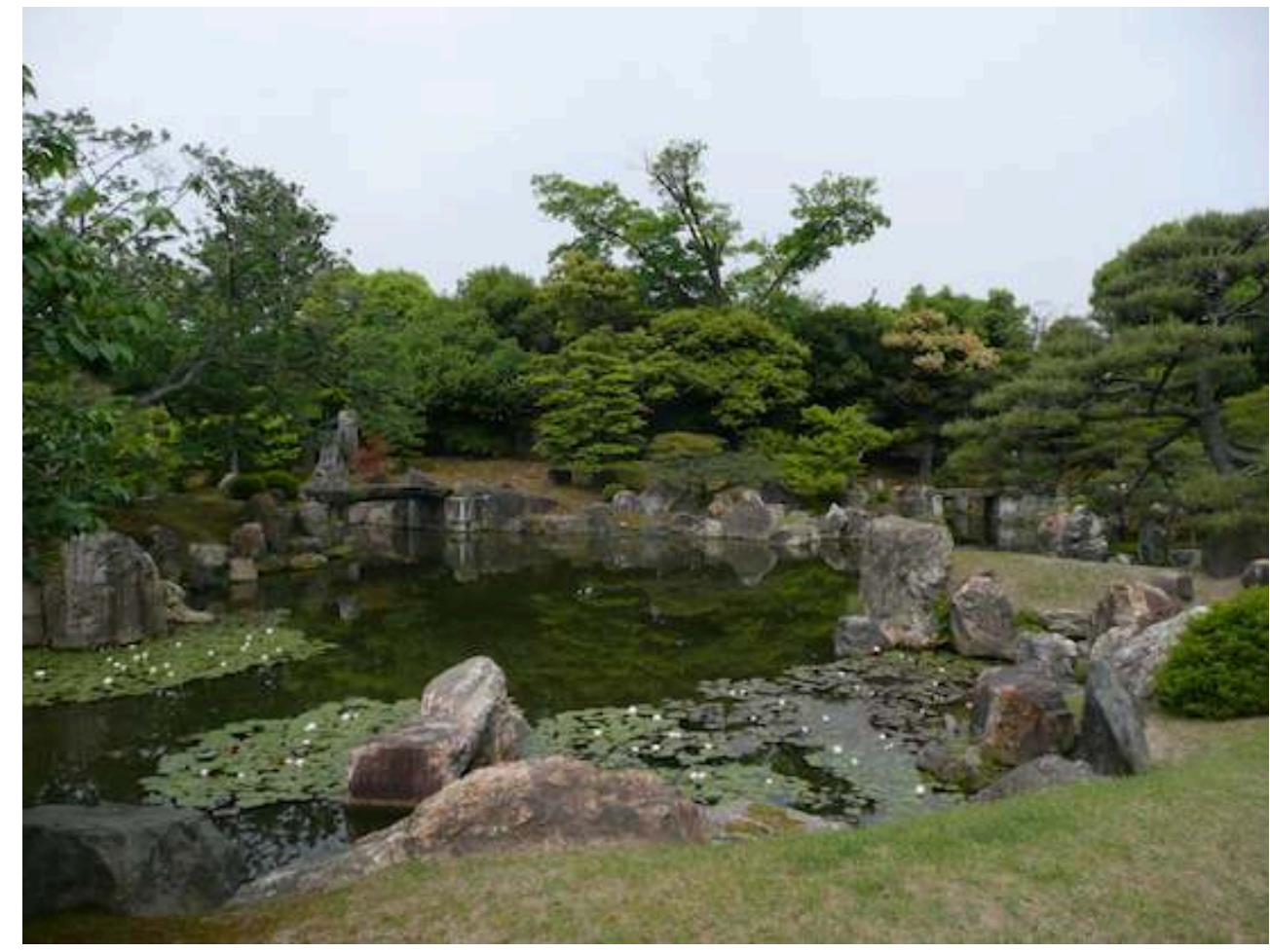

D’après Mori Osamu, lorsque Kobori Enshū a conçu sur cette berge le palais pour la venue de l'empereur au château en 1626, il aurait arrangé la disposition des pierres pour mettre en valeur ce côté du jardin.

Source : Emmanuel Marès. 
Figure 11. Étang du château de Nijō depuis le palais du shogun au nord

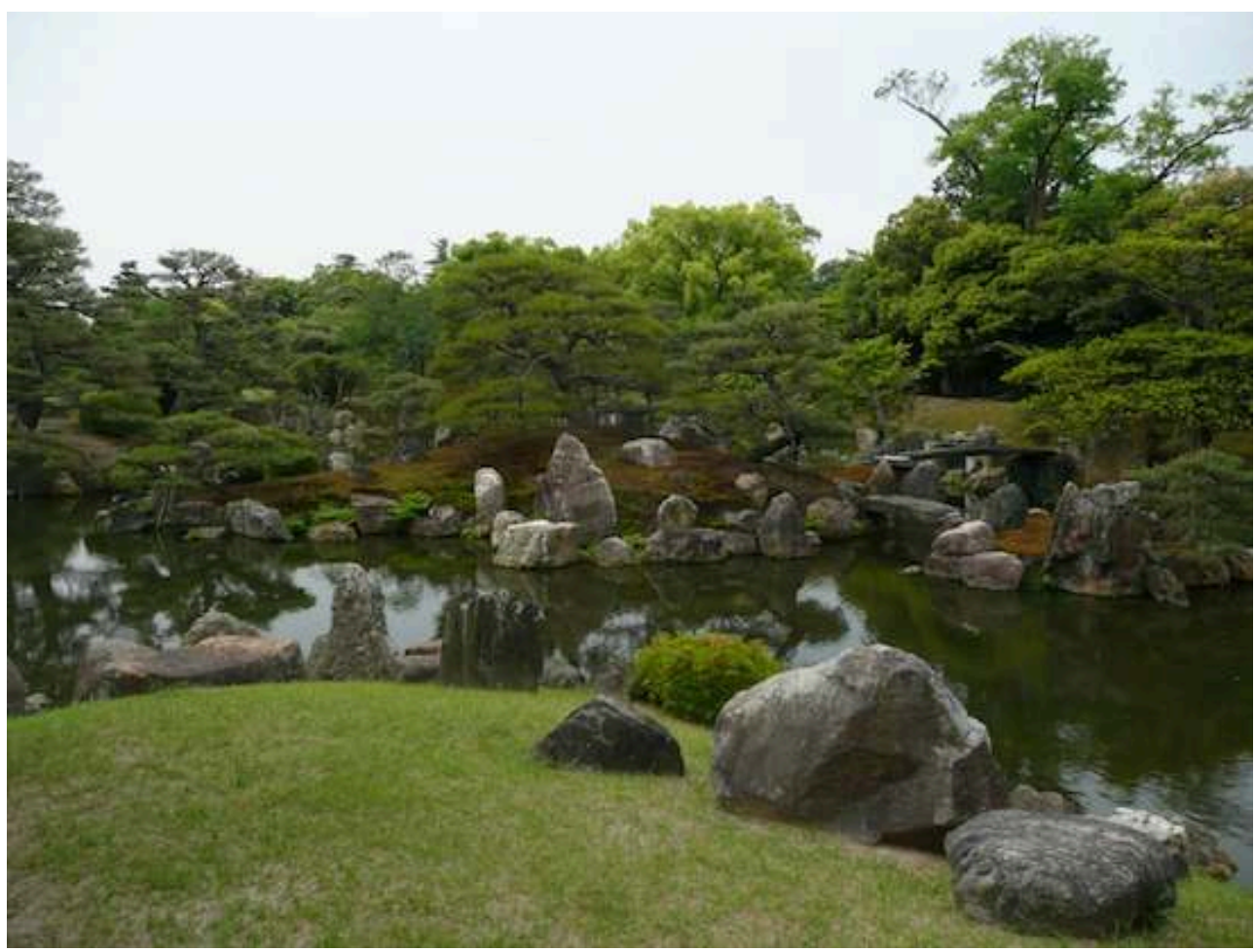

Source : Emmanuel Marès.

officielles, et il sera logiquement désigné comme responsable des travaux d'aménagement pour la venue de l'empereur Gomizuno.o 後水尾天皇 (1596-1680) le septième mois de la même année. Il va dessiner les plans du bâtiment qui sera construit au sud de l'étang pour recevoir l'empereur. À cette occasion, Enshū aurait pris soin de réorienter les pierres du jardin de telle sorte qu'on puisse le contempler à la fois depuis le palais du shogun, situé au nord, et depuis le palais de l'empereur, situé au sud. Du moins, c'est ainsi que se présente la thèse de Mori Osamu.

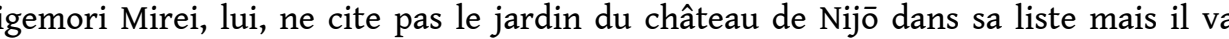
quand même aborder ce sujet un peu plus loin (p. 93-98). En ce qui concerne le jardin, et tout particulièrement les arrangements de pierres tels qu'ils ont été conservés jusqu'à aujourd'hui, Shigemori Mirei y voit le reflet du travail de Kentei 賢庭 (dates inconnues), jardinier renommé de l'époque qui a travaillé notamment sur la conception du jardin du temple Sanbō-in 三宝院 et sous la direction de Kobori Enshū pour le jardin $\mathrm{du}$ temple Konchi-in. Mais aucune source historique ne faisant allusion à une quelconque intervention de Kobori Enshū sur le jardin, Shigemori Mirei préfère ne pas prendre parti.

(3) D'après Mori Osamu, le jardin du pavillon principal du temple Nanzen-ji aurait été commandé en 1629 à Kobori Enshū par Ishin Sūden 以心崇层 (1569-1633), supérieur du temple Konchi-in, annexe du Nanzen-ji. 
Figure 12. Le jardin sud du pavillon principal du temple Nanzen-ji

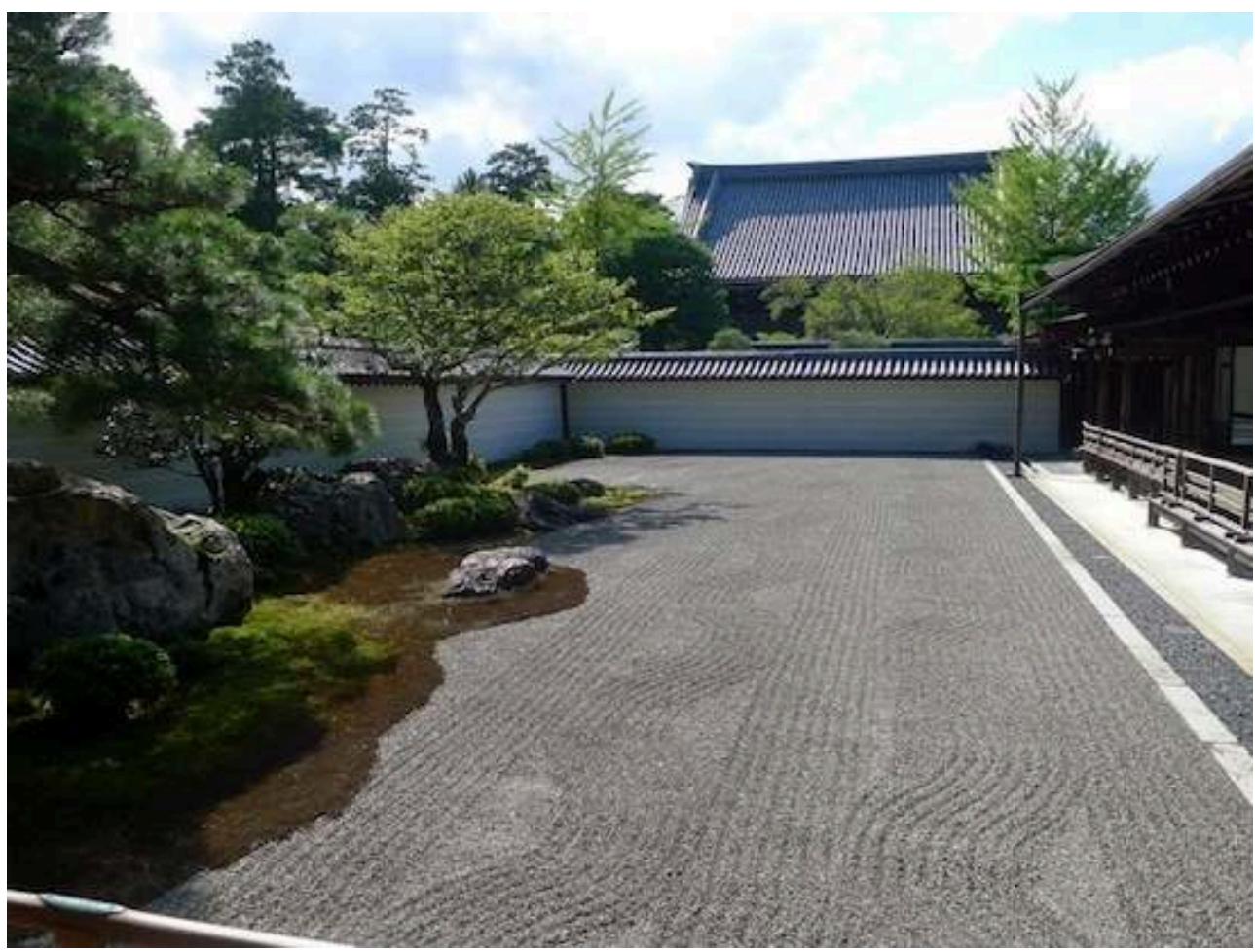

Une composition très simple : les pierres sont alignées au fond du jardin, laissant une grande partie de l'espace vide. Des vagues sont dessinées dans le gravier.

Source : Ursula Wieser. 
Figure 13. Vue panoramique du jardin du Nanzen-ji

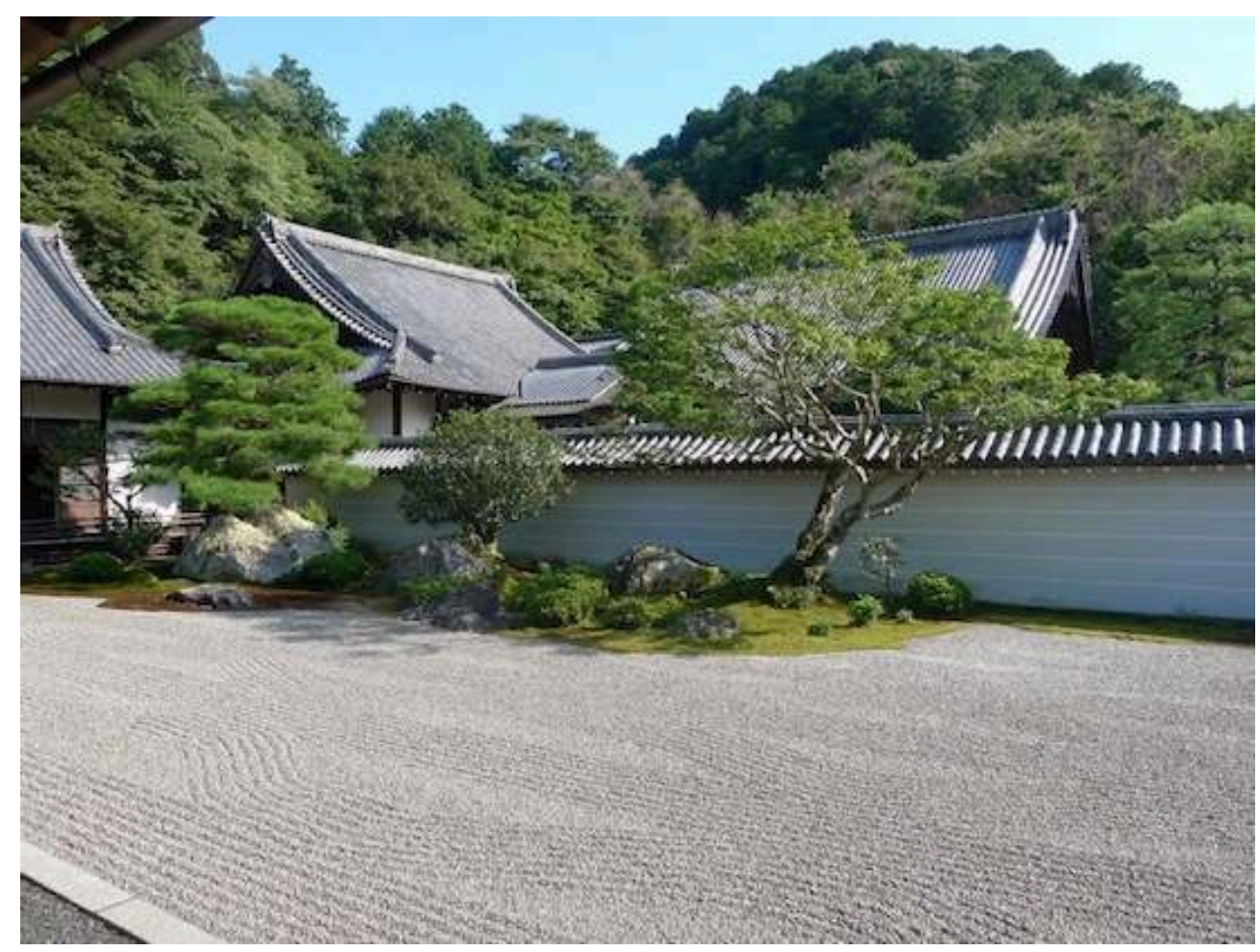

La courbe que dessinent les pierres rappelle la forme de la colline juste derrière le temple.

Source: Ursula Wieser.

47 Mori Osamu s'appuie sur la note du $26^{\mathrm{e}}$ jour du onzième mois de 1629 du journal d'Ishin Sūden, le Honkō kokushi nikki 本光国師日記, où il est effectivement question du « jardin de Nanzen-ji ». Mais alors que les travaux du jardin du temple Konchi-in sont décrits avec beaucoup de précision, depuis la commande en 1628 jusqu'à la réalisation en 1633, il n'y a qu'une seule note concernant ce «jardin de Nanzen-ji ». De plus, le pavillon principal n'est jamais mentionné, seul le terme de Nanzen-ji apparaît. Dans ces conditions, ne serait-il pas plus juste de penser que lorsque Ishin Sūden fait allusion au «Nanzen-ji », il veut parler du temple annexe qui est le sien, le Konchi-in? Ce qui signifierait qu'il ne s'agit pas d'une nouvelle commande mais plutôt d'une reformulation de sa requête exprimée un an auparavant pour le jardin du temple Konchi-in.

Il semblerait que Mori Osamu extrapole un peu vite. Qu'en pensait Shigemori Mirei ? Dans son livre, il n'a pas relevé ce détail et ne fait jamais allusion au jardin du pavillon principal du temple Nanzen-ji.

49 (4) Mori Osamu donne le plan du palais Shin.in gosho, qui date de 1640 (p. 195). Kobori Enshū a travaillé sur ce projet dans le cadre de sa fonction officielle. Malheureusement, ce palais a brûlé et il ne reste plus aucun vestige. Dans sa chronologie, Shigemori Mirei note bien que Kobori Enshū a travaillé en tant que maître d'œuvre des constructions officielles sur le projet du Shin.in gosho mais il ne l'inscrit pas dans sa liste de jardins et ne lui consacre aucune ligne. Comment interpréter ce silence? Peut-être par un manque d'informations, il est probable que Mirei n'ait pas eu connaissance de ce plan conservé dans les archives impériales et donc difficiles d'accès. 
50 (5) Enfin, vient le problème de la villa Katsura, déjà évoqué plus haut. Cette résidence a été construite en deux temps : les premiers travaux ont été commandés par Hachijō no miya Toshihito 八条宮智仁 (1579-1629). Il s'agissait alors d'une petite résidence secondaire située au sud-ouest de Kyōto, au bord de la rivière Katsura. C'est son fils, Hachijō no miya Toshitada八条宮智忠 (1619-1662) qui va poursuivre les travaux et faire construire les bâtiments et le jardin qui ont traversé le temps et qui restent une source d'inspiration pour les architectes et les paysagistes d'aujourd'hui.

Figure 14. Une des vues les plus connues de la villa Katsura

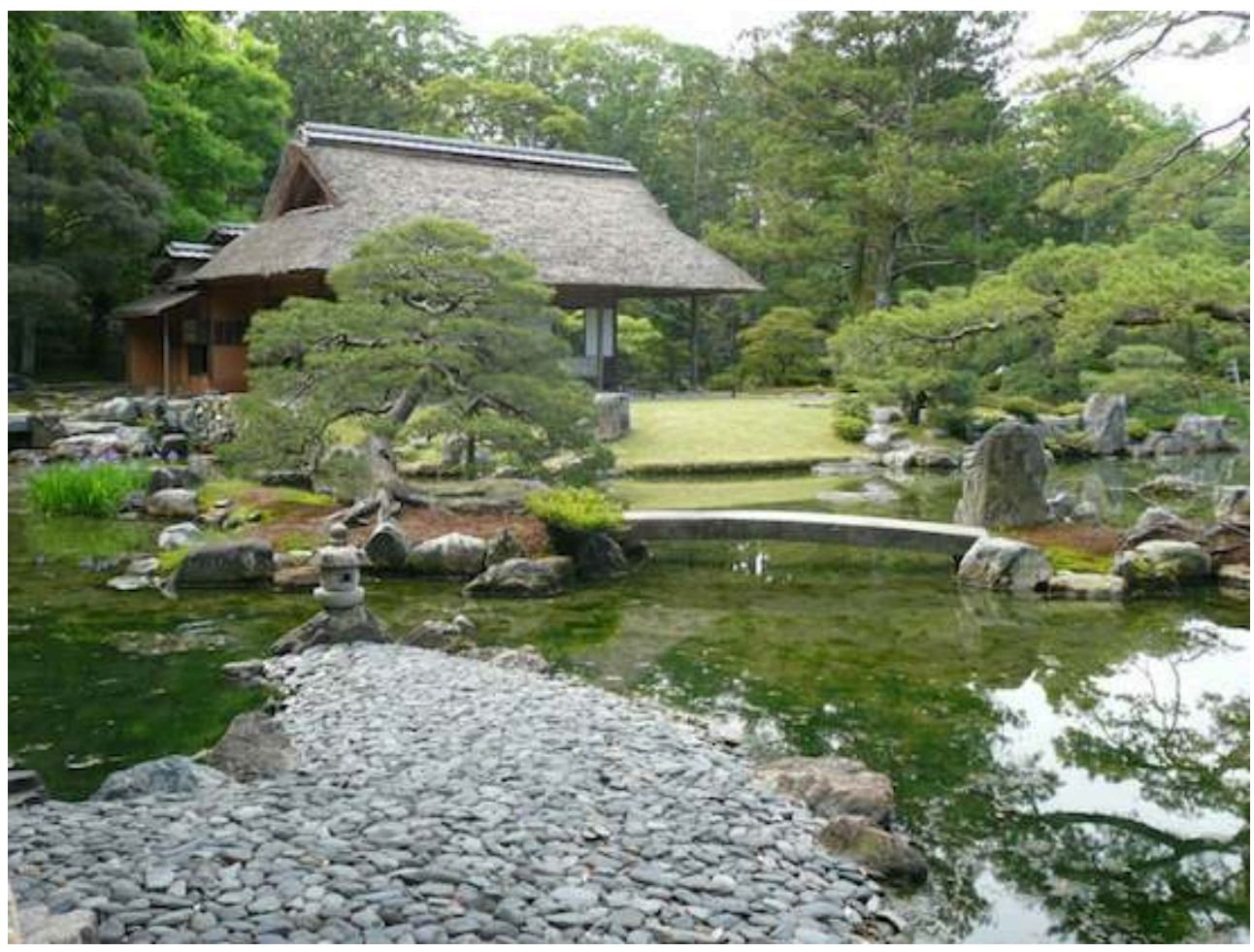

Une plage de galet avec une petite lanterne et, de l'autre côté de l'étang, le pavillon Shōkin-tei.

Source : Emmanuel Marès. 
Figure 15. Le décor en damier à l'intérieur du pavillon Shōkin-tei

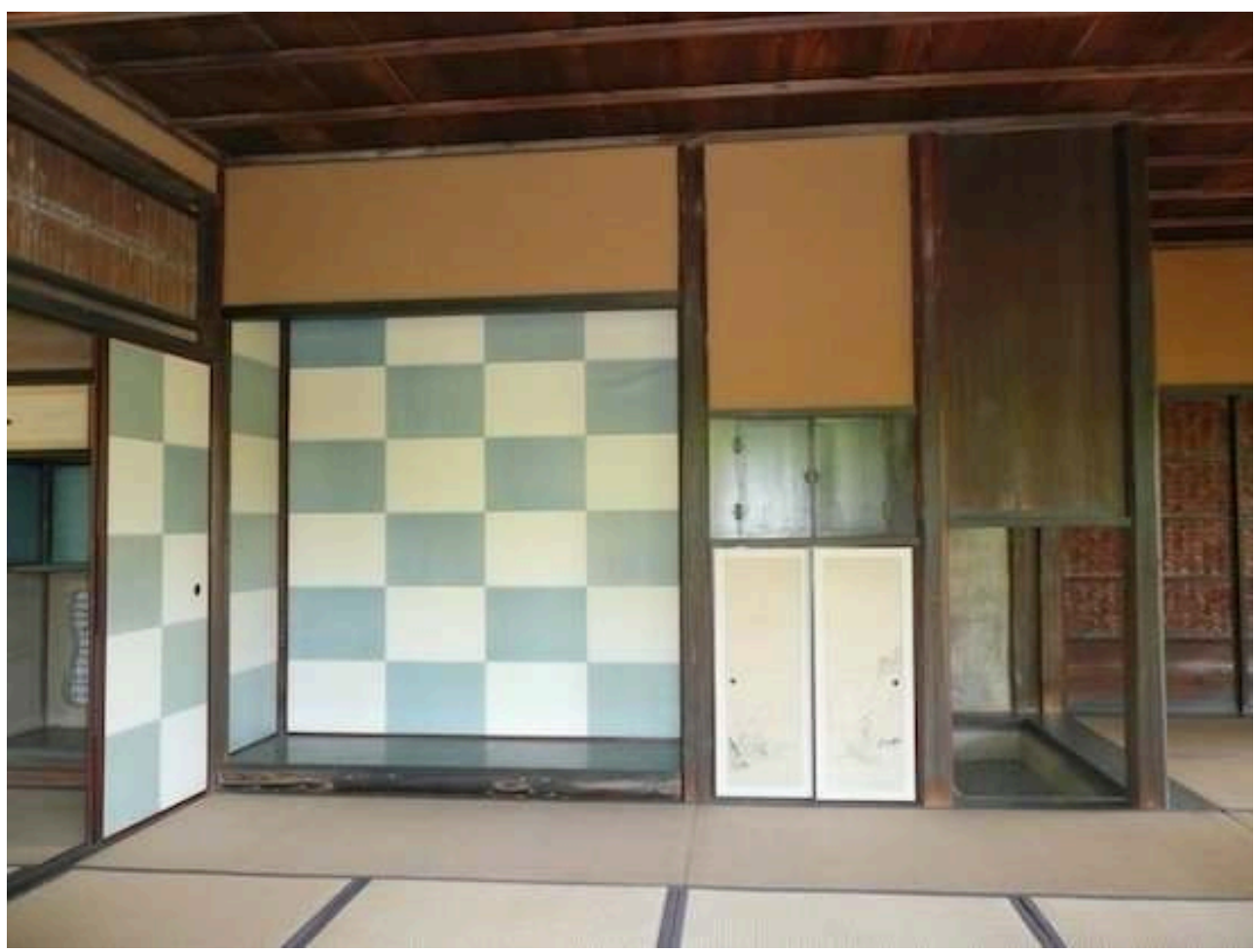

Shigemori Mirei s'est probablement inspiré de ce motif pour le jardin du temple Tōfuku-ji. Source : Emmanuel Marès. 
Figure 16. Pas japonais dans le jardin de la villa Katsura

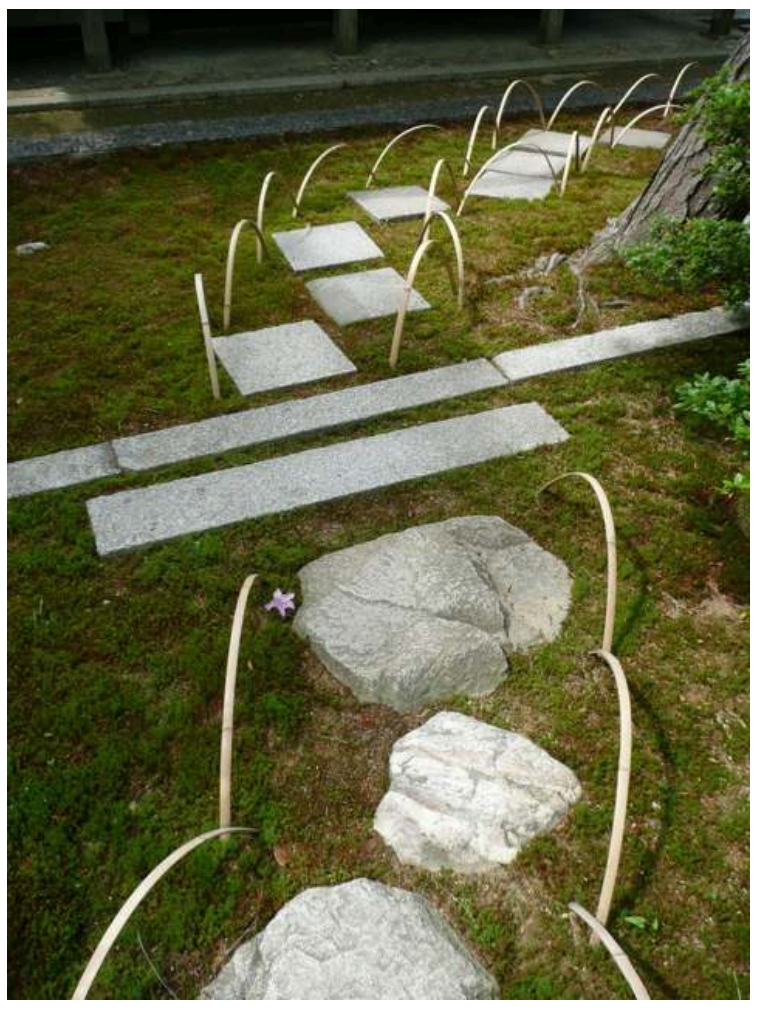

Une frontière dans l'espace est clairement marquée par l'opposition entre pierres naturelles et pierres taillées.

Source : Emmanuel Marès.

Figure 17. Le palais Katsura, un chef-d'œuvre de l'architecture dite sukiya-zukuri

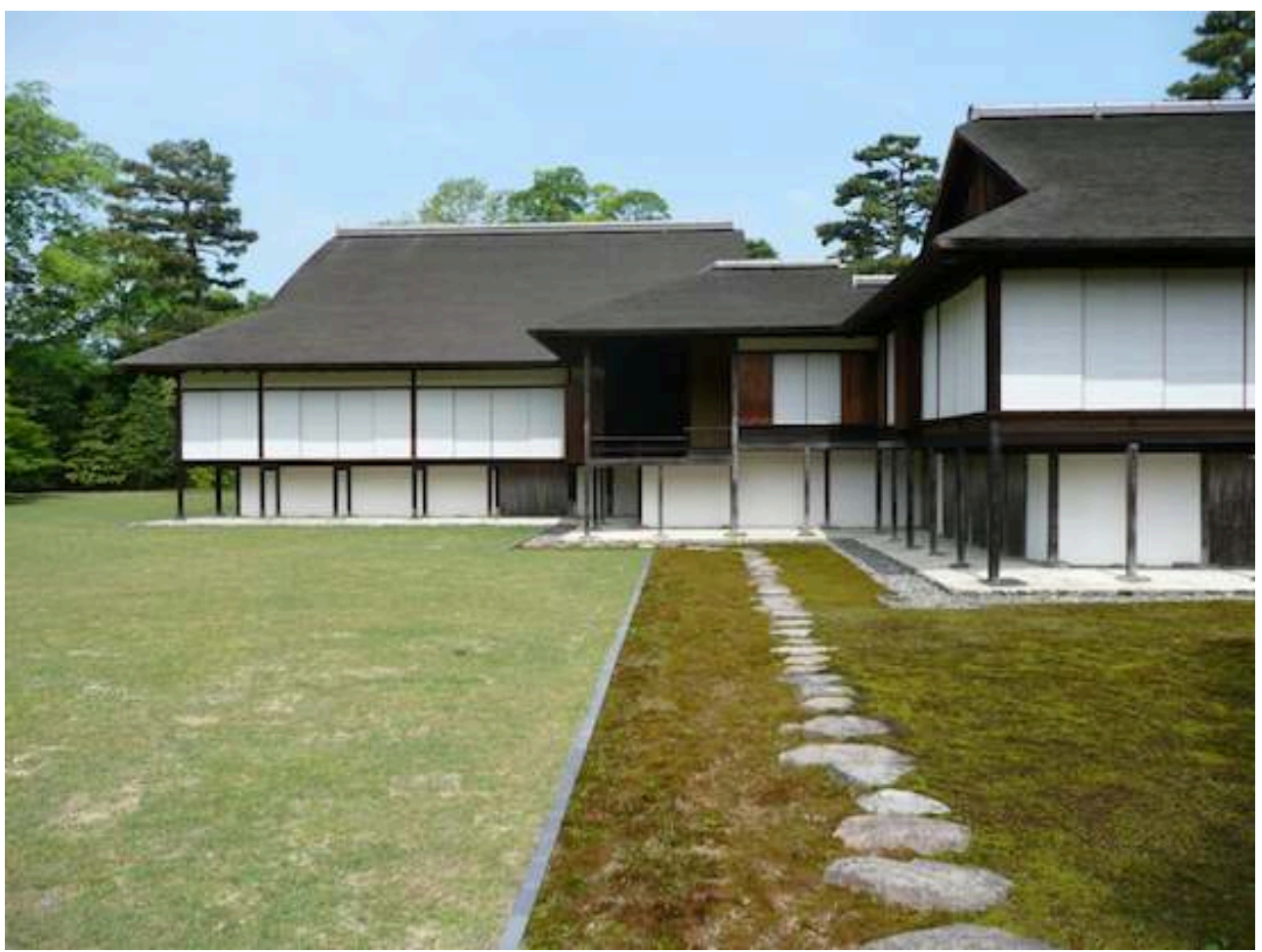

Source : Emmanuel Marès. 
51 Pour Shigemori Mirei, Kobori Enshū aurait dirigé les seconds travaux à partir de 1641. Il se base sur un document, Katsura gobetsugyō no koto 桂御別業之事 (Choses concernant la résidence secondaire Katsura), qui a été écrit a posteriori. Il est bien conscient de ce problème mais, tout comme pour le cas du temple Raikyū-ji, il ne remet pas en question cette source. Selon lui, un tel chef-d'œuvre ne pourrait être le résultat du travail de quelqu'un d'autre.

Mori Osamu, qui a consacré plusieurs ouvrages à la villa Katsura et à son histoire, affirme que Kobori Enshū n'a pas pu prendre part à la conception de cet immense projet. En se lançant dans des études poussées sur ce sujet épineux de l'histoire des jardins japonais, il s'était fait un point d'honneur d'élucider cette question et de mettre fin aux débats, un objectif qu'il a atteint puisque, aujourd'hui, sa thèse prévaut dans le milieu universitaire et est aussi largement diffusée auprès du grand public.

Lorsqu'il écrit la biographie de Kobori Enshū en 1967, Mori Osamu a déjà exposé ce problème à plusieurs reprises et il évite la redondance. Il n'évoque cette question que deux fois, mais à des endroits clés : dans la préface tout d'abord, où il dit que cela a été le point de départ de ses recherches ; et à la dernière page enfin, lorsqu'il est question de la descendance d'Enshū. Le fait que le livre s'ouvre et se clôt sur ces propos montre à quel point cette question devait obnubiler Mori Osamu et, sans aucun doute, tous ses contemporains.

\section{Pour aller au-delà de la simple opposition}

Quelle synthèse faire de ces deux analyses? Aucun document historique susceptible d'éclaircir les interrogations que nous venons de soulever n'a été mis à jour depuis. De toute façon, notre démarche ne consiste pas à chercher une vérité ou à donner raison à une théorie pour discréditer l'autre. Il s'agit plutôt de comprendre le regard que ces deux hommes portaient sur les jardins et leur histoire. Comment et pourquoi arriventils à des conclusions divergentes?

En effet, à l'exception du cas du Shin.in gosho, pour lequel il semblerait que Shigemori Mirei n'ait pas eu accès aux mêmes sources que Mori Osamu, les deux auteurs se basent sur les mêmes documents pour défendre des thèses qui s'opposent. À la lecture de ces deux livres, il semble donc possible d'esquisser deux attitudes, deux orientations, deux tendances représentatives de la conception de l'histoire des jardins japonais.

Que ce soit pour le jardin du temple Raikyū-ji ou pour la villa Katsura, Shigemori Mirei donne raison à des archives qui ont été écrites a posteriori. Mirei était bien conscient de ce problème qui fragilisait considérablement son raisonnement. Il en parle ouvertement dans son livre et accepte les critiques (p.71) et pourtant, ce sont des thèses qu'il a défendues envers et contre tout jusqu'à la fin de sa vie.

Que cherchait-il à prouver ainsi ? Voulait-il croire à tout prix à l'histoire ancienne? Présenter la villa Katsura comme étant l'œuvre de Kobori Enshū était peut-être une façon de rendre hommage au plus fameux des paysagistes japonais. En effet, la villa Katsura est l'un des rares exemples de l'époque qui ait été bien conservé et qui soit toujours parfaitement entretenu. C'est un lieu emblématique à tout point de vue. Pour Shigemori Mirei, dire, comme tous les spécialistes s'accordent à le penser aujourd'hui, qu'il ne s'agit que du reflet de l'esthétique mise au point par Enshū à cette époque ne 
suffit pas. Est-ce qu'il faut en déduire que pour lui, sans la villa Katsura, le mythe de Kobori Enshū s'effondrait?

De même, en attribuant le jardin du temple Raikyū-ji au jeune Kobori Enshū, peut-être voulait-il montrer que celui-ci avait un don inné pour l'art des jardins. Ou alors, était-il important pour lui qui venait de cette région de penser qu'un autre paysagiste de génie l'avait précédé ? Lorsqu'il dit dans sa préface qu'il se sent profondément lié au destin d'Enshū parce qu'il est né non loin du jardin de Raikyū-ji, n'essaye-t-il pas de s'approprier le personnage, et même de s'y identifier ? À ce propos, un passage du livre est éloquent. Dans le quatrième chapitre, lorsqu'il évoque «l'esprit et la forme du thé selon Enshū », Shigemori Mirei écrit :

« [...] Il [Enshû] croyait avant tout au pouvoir de l'être humain, au fait que l'action humaine, en contrôlant toutes les choses, pouvait reconstruire la nature. D'un point de vue artistique, lorsqu'il s'agit de représenter la nature, [Enshū] croyait qu'il n'y avait pas besoin d'imiter la nature ou de s'y conformer sans réserve. Selon la créativité du concepteur, il avait la certitude qu'il était possible de recréer la nature dans toute sa grandeur ou même de la redécouvrir. Tant que l'on n'a pas perçu cela, certains aspects de l'art d'Enshū sont difficilement compréhensibles ${ }^{15}$. »

Peu importe de savoir si cette analyse convient à décrire l'esthétique d'Enshū ou non, ce qui est certain, c'est qu'un spécialiste aujourd'hui pourrait tenir le même discours au sujet des jardins conçus par Shigemori Mirei. Son but n'était pas de faire un jardin qui ressemble à la nature. Il refusait le mimétisme et cherchait à insuffler dans ses œuvres quelque chose de surnaturel et même de divin. Il est évident que Mirei voyait dans les réalisations d'Enshū l'idéal qu'il a cherché à atteindre toute sa vie : « l'éternel moderne (eien no modan 永遠のモダン)».

D'ailleurs, ce n'est sûrement pas un hasard si Mirei a lui aussi beaucoup pratiqué la cérémonie du thé. Il s'était affranchi de toutes les différentes écoles et il avait réussi à développer son propre style. Pour le nouvel an, il recevait des moines, des artistes, des professeurs, au total plus de deux cents personnes se succédaient sur quelques jours pour assister à la grande cérémonie du thé qu'il organisait périodiquement dans sa maison à Kyōto.

Difficile de ne pas faire le rapprochement avec Enshū qui a su se démarquer de ses maîtres pour développer une esthétique qui lui était propre. De plus, tout au long de sa vie, il a organisé des cérémonies du thé avec tous les moines, artistes et autres acteurs culturels les plus importants de son époque. Il est donc permis de penser que pour Mirei, Enshū était comme une icône à laquelle il a essayé de s'identifier, l'incarnation de l'artiste complet que lui-même aspirait à devenir. Si l'on pousse le raisonnement jusque-là, on peut se poser des questions sur les motivations de Shigemori Mirei : lorsqu'il écrit la biographie de Kobori Enshū, n'est-il pas en train de faire un pamphlet pour défendre sa propre vision de l'art du jardin? Sous couvert de décrire l'art de ce personnage mythique, n'est-il pas en train de justifier sa version de l'histoire des jardins et son attitude avant-gardiste en tant que créateur? Peut-être que les critiques qu'il a dû essuyer de son vivant, et venant tout particulièrement du milieu académique, étaient suscitées par sa tendance à s'approprier l'histoire ?

L'analyse de Mori Osamu, notamment en ce qui concerne les jardins du temple Raikyūji et de la villa Katsura, semble se baser sur une méthode sinon plus objective, au moins plus scientifique. Il ne cherche pas à attribuer coûte que coûte ces deux jardins à Kobori Enshū. L'examen des documents d'époque et de ceux qui ont été écrits a posteriori montre bien que les probabilités pour qu'Enshū soit effectivement le concepteur de ces 
jardins sont faibles, voire quasi nulles. Ces résultats poussent donc Mori Osamu à aller à l'encontre de la tradition. Il fait tomber l'icône de son piédestal.

Et pourtant, nous avons vu plus haut que ses conclusions concernant les jardins du château de Nijō et du temple Nanzen-ji sont très approximatives. Dans ces deux cas, il délaisse sa démarche scientifique et se base sur son intuition ou sur une simple supposition. Qu'il émette une hypothèse, pourquoi pas? Mais qu'il présente des faits qui ne sont pas fondés sans émettre le moindre doute, voilà qui est plus étonnant. Lui qui s'est toujours montré si méfiant vis-à-vis de la légende qui entoure Enshū, lui qui a toujours voulu faire parler la raison avant l'imagination, on a l'impression que, soudain, il baisse sa garde. N'aurait-il pas brisé un mythe pour essayer d'en construire un autre? Ou bien faut-il voir ici une façon de se démarquer du travail de Shigemori Mirei ?

Dans son analyse des travaux du château de Nijō, Shigemori Mirei se montre plus prudent que Mori Osamu et, s'il évoque effectivement une possible intervention de Kobori Enshū qui était chargé de dessiner un bâtiment au bord de l'étang, il ne tire pas de conclusion hâtive à propos de l'aménagement du jardin. Au contraire, Mori Osamu insinue qu'Enshū, architecte mais aussi paysagiste de génie, ne pouvait pas travailler sur un bâtiment sans penser à l'agencement du jardin pour créer un ensemble harmonieux. Il s'agit là, en réalité, d'une des idées qui sert de fil conducteur dans le livre de Mori Osamu. Mais si l'exemple semble venir parfaitement à propos, aucun texte historique ne permet de soutenir une telle interprétation. Alors, comment ne pas émettre des doutes sur le bien-fondé de cette démarche qui consiste à présenter une simple intuition comme un fait établi ?

Pour ce qui est du pavillon principal du temple Nanzen-ji, Shigemori Mirei n'y fait même pas allusion. Il semblerait que l'hypothèse selon laquelle Kobori Enshū aurait conçu ce jardin ne lui ait même pas traversé l'esprit. Ce qui est troublant quand on avance dans cette analyse c'est que le jardin du temple Raikyū-ji, avec ses pierres dressées, ses arbustes taillés et ses symboles, correspond parfaitement à l'esthétique de Shigemori Mirei. Le jardin du pavillon principal du temple Nanzen-ji, quant à lui, reflète à merveille la sensibilité de Mori Osamu: ce sont des pierres imposantes disposées de manière discrète. Elles ne sont pas là pour donner un sens particulier mais uniquement comme un élément de la composition. De même, les végétaux ne sont pas sculptés pour représenter des vagues ou autre chose, ils sont là pour ce qu'ils sont.

Alors que Shigemori Mirei présentait l'art de Kobori Enshū comme une mainmise sur la nature, Mori Osamu propose l'analyse inverse :

« Les jardins d'Enshū étaient avant tout des adaptations de la nature. Le livre secret du Sakutei-ki de l'époque de Heian insistait déjà sur ce point et Enshū excellait dans l'utilisation des paysages naturels, l'idéal des concepteurs de jardins de l'époque de Heian $^{16}$. »

67 Finalement, Enshū était-il plutôt du côté de l'artifice comme le dit Shigemori Mirei ou alors du côté de la nature comme le dit Mori Osamu ? Ce sont là deux visions du jardin, deux idéaux qui s'opposent. Pour Shigemori Mirei, c'est l'acte créateur qui est important, la main du paysagiste ne doit rien laisser au hasard. Le jardin est un espace qui interroge l'homme sur son rapport à la nature et au divin. C'est pourquoi on ne peut pas se contenter de faire une pâle copie ou une simple représentation de la nature. Chaque élément entrant dans la composition d'un jardin est doté d'un sens profond. 


\section{BIBLIOGRAPHIE}

Horiguchi, Sutemi, 堀口捨己, «Kobori Enshū no gazō 小堀遠州の画像 (Les portraits de Kobori Enshū », dans Shisō思想 (Pensée), Tōkyō, Iwanami shoten 岩波書店, décembre 1934.

Horiguchi, Sutemi, (éd.), Chashitsu okoshiezu 茶室おこし絵困集 (Recueil de plans de pavillons de thé en 3 dimensions), Tōkyō, Bokusui shobō 墨水書房, 1964.

Kobori, Sōjitsu (supervision), Daimyo Tea Master : Kobori Enshū, artistic encounters - Celebrating Kobori Enshū’s 400-year Anniversary, Tōkyō, Asahi shinbunsha, 2007, 196 p.

Kobori, Sōjitsu, 小堀 宗慶, Kobori Enshû no bi wo tazunete 小堀遠州の美を訪祊て (À la rencontre de l'esthétique de Kobori Ensh̄̄), Tōkyō, Shūeisha 集英社, 2010, 208 p.

Mori, O. 森蓝, Heian jidai no teien no kenkyū 平安時代の庭園の研究 (Étude sur les jardins de l'époque de Heian), Tōkyō, Kuwana bunseidō 桑名文星堂, 1945.

Mori, Osamu, Katsura rikyū no kenkyū 桂離宮の研究 (Étude sur la villa Katsura), Tōkyō bunkasha 東 都文化社, 1955.

Mori, Osamu, Katsura rikȳ̄ 桂離宮 (La villa Katsura), Tōkyō, Shūeisha 集英社, 1967.

Mori, Osamu, Niwa hitosuji『庭ひとすじ』(Une vie consacrée au jardin), Tōkyō, Gakuseisha 學生社, 1975,245 p.

Mori, Osamu, Kobori Enshū no sakuji 小堀遠州の作事 (L’Euvre de Kobori Enshū), Tōkyō, Kichikawa kōbunkan 吉川弘文館, Nara kokuritsu bunkazai kenkyūjo gakuhō jyūhachisatsu 奈良国立文化財 研究所学報第十八冊 (18 rapport du Centre de recherche national de Nara pour les propriétés culturelles), mars 1967. 
Sawashima, Eitarō, 沢島英太郎, Kobori Enshū no kenkȳu 小堀遠州の研究 (Étude sur Kobori Enshū), Osaka, Sōgensha 大阪創元社, 1937.

Sawashima, Eitarō, « Kohō-an sho.in oyobi chashitsu kenkyū 孤蓬庵書院及び茶室の研究 (Étude sur le pavillon principal et du pavillon de thé du temple Kohō-an) », dans Shiseki to bijutsu 史迹と 美術 (Vestiges et art), Tōkyō, Suzukake shuppanshaスズカケ出版社, avril-mai 1938.

Sawashima, Eitarō, « Konchi-in Enshū saku chashitsu ni tsuite 金地院遠州作茶室について (À propos du pavillon de thé conçu par Enshū dans le temple Konchi-in) », dans Gasetsu 画説, Tōkyō, Tōkyō bijutsu kenkyūjō 東京美術研究所, mai 1940.

Sawashima, Eitarō, « Ryōkō-in Mittan no seki ni tsuite 竜光院密庵の席に就いて (À propos du pavillon de thé Mittan dans le temple Ryōkō-in) », dans Gasetsu 画説, Tōkyō, Tōkyō bijutsu kenkyūjōo 東京美術研究所, avril 1941.

Shigemori, Mitsuaki, 重森三明, Shigemori Mirei II 重森三玲2, Kyōto, Tsūshinsha, 2010, 120 p.

Shigemori, Mirei, 重森三玲, Nippon teien shi zukan 日本庭園史困鑑 (L'histoire illustrée des jardins japonais), Tōkyō, Yūkōsha 有光社, 1936-1939, 26 vol.

Shigemori, Mirei重森三玲, Shigemori Kantō 重森完途, Nihon teien shi taikei 日本庭園史体系 (Histoire systématique des jardins japonais), Tōkyō, Shakai shisōsha 社会思想社, 1971-1976, 35 vol.

Shigemori, Mirei, 重森三玲, « Shinsakutei-ki 新作庭記 (Nouveau traité sur la conception des jardins) ", Niwa - kamigami he no apuro-chi shigemori mirei sakuhinshū 庭一神々へのアプローチ 重森三玲作品集 (Le Jardin, une approche du divin, œuvres de Shigemori Mirei), Tōkyō, Seibundō shinkōsha 誠文堂新光社 1976, p. 281-315 p.

Tamura, Tsuyoshi, 田村剛, « Karesansui to Shigemorishi no shojyosaku 枯山水と重森氏の処女 作 (Le jardin sec et la première œuvre de M. Shigemori) », Teien 庭園 (Jardins), Tōkyō, juin 1925.

\section{Dictionnaires et autres}

Nedan no (Meiji/Taishō/Shōwa) fūzokushi 值段の〈明治 · 大正 · 昭和〉風俗史 (Histoire des mœurs et des prix, Meiji/Taishō/Shōwa), Tōkyō, Asahi shinbunsha, 朝日新聞社, 1987, 620 p.

Zōen yōgo jiten 造園用語辞典 (Dictionnaire des termes paysagers) (1985), Tōkyō, Shōkokusha 彰国社, 2011, 535 p.

\section{NOTES}

1. L'exposition « Daimyo Tea Master : Kobori Enshū, artistic encounters (Kobori Enshū bi no deai ten 小堀遠州 美の出会い展) », organisée par la Compagnie Asahi Shinbun s'est déroulée de décembre 2007 à mars 2008. Pendant cette période, elle a été présentée dans la galerie de Ginza Matsuya à Tōkyō du 30 décembre 2007 au 14 janvier 2008 (95 199 personnes en 16 jours), au musée Matuzakaya à Nagoya du 23 février au 16 mars (29 213 personnes en 23 jours) et enfin au musée Daimaru à Kobe du 19 mars au 31 mars 2008 (18 736 personnes en 13 jours). On peut consulter à ce sujet le catalogue de l'exposition : Kobori Sōjitsu (supervision), Daimyo Tea Master : Kobori Enshū, artistic encounters - Celebrating Kobori Enshū's 400-year Anniversary, Tōkyō, Asahi shinbunsha, 2007, 196 p. (Les chiffres présentés ici sont les chiffres officiels fournis par la Compagnie Asahi Shimbun)

2. La maison familiale de Shigemori Mirei n'existe plus mais le pavillon de thé Tenrai-an a été démantelé et reconstruit dans l'enceinte du sanctuaire shinto Yoshikawa Hachiman-gū en 1969. Cf. Shigemori, Mitsuaki, 重森三明, Shigemori Mirei II 重森三玲2, Kyōto, Kyōto Tsūshinsha, 2010, $120 \mathrm{p}$. 
3. Shigemori, Mirei, 重森三玲, Nippon teien shi zukan 日本庭園史困鑑 (L'Histoire illustrée des jardins japonais), Tōkyō, Yūkōsha 有光社, 1936-1939, 26 vol. À la fin de sa vie, il a publié une version révisée et augmentée de cette étude avec l'aide de son fils aîné : Shigemori Mirei 重森三玲, Shigemori Kantō 重森完途, Nihon teien shi taikei 日本庭園史体系 (Histoire systématique des jardins japonais), Tōkyō, Shakai shisōsha 社会思想社, 1971-1976, 35 vol.

4. Cf. «Shinsakutei-ki 新作庭記 (Nouveau traité sur la conception des jardins) » dans Shigemori, Mirei, 重森三玲, Niwa - kamigami he no apuro-chi shigemori mirei sakuhinshū 庭一神々へのアプロー 于 重森三玲作品集 (Le Jardin, une approche du divin, ceuvres de Shigemori Mirei), Tōkyō, Seibundō shinkōsha 誠文堂新光社 1976, p. 281-315 pages. Dans cet essai écrit à la fin de sa vie et qui résume parfaitement la vision du jardin de Shigemori Mirei, il souligne à plusieurs reprises le fait que le jardin est un «art » et, qu'à ce titre, une œuvre d'art ne peut être le résultat du travail d'un artisan mais d'un artiste.

5. Mori, Osamu, 森蘊, Niwa hitosuji『庭ひとすじ』(Une vie consacrée au jardin), Tōkyō, Gakuseisha 學生社, , 1975, 245 p.

6. Tamura, Tsuyoshi, 田村剛, «Karesansui to Shigemorishi no shojyosaku 枯山水と重森氏の処女作 (Le jardin sec et la première œuvre de M. Shigemori) », dans Teien 庭園 (Jardins), Tōkyō, juin 1925. 7. Mori, Osamu, 森緧, Heian jidai no teien no kenkyū 平安時代の庭園の研究 (Étude sur les jardins de l'époque de Heian), Tōkyō, Kuwana bunseidō 桑名文星堂, 1945.

8. Sotoyama Eisaku a été diplômé de l'université impériale de Tōkyō en 1916. Ensuite il est devenu professeur dans cette même institution. Sur ce sujet, voir Zōen yōgo jiten 造園用語辞典 (Dictionnaire des termes paysagers) (1985), Tōkyō, Shōkokusha彰国社, 2011, 535 p.

9. Ces informations ont été obtenues auprès de Nakamura Masao, actuel directeur général des éditions Kawahara Shoten et de l'Association de l'architecture et des techniques traditionnelles de Kyōto (Kyōto dentō kenchiku gijutsu kyōkai京都伝統建築技術協会), qui a connu Shigemori Mirei en personne et que je voudrais remercier ici pour avoir pris le temps de répondre à mes questions.

10. Horiguchi, Sutemi, 堀口捨己, «Kobori Enshū no gazō 小堀遠州の画像 (Les portraits de Kobori Enshū », dans Shisō 思想 (Pensée), Tōkyō, Iwanami shoten 岩波書店, décembre 1934. Ou encore, sous la direction de Horiguchi Sutemi, Chashitsu okoshiezu 茶室おこし絵龱集 (Recueil de plans de pavillons de thé en 3 dimensions), Tōkyō, Bokusui shobō 墨水書房, 1964.

Sawashima, Eitarō, 沢島英太郎, Kobori Enshū no kenkyū 小堀遠州の研究 (Étude sur Kobori Enshū), Osaka, Sōgensha 創元社, 1937. Du même auteur : «Kohō-an sho.in oyobi chashitsu kenkyū 孤蓬 庆書院及び茶室の研究 (Étude sur le pavillon principal et du pavillon de thé du temple Kohōan)», dans Shiseki to bijutsu 史迹と美術 (Vestiges et art), Tōkyō, Suzukake shuppanshaスズカケ出 版社, avril-mai 1938. 《Konchi-in Enshū saku chashitsu ni tsuite 金地院遠州作茶室について (À propos du pavillon de thé conçu par Enshū dans le temple Konchi-in) », Gasetsu 画説, Tōkyō bijutsu kenkyūjō東京美術研究所, mai 1940. «Ryōkō-in Mittan no seki ni tsuite 竜光院密庵の席に 就いて (À propos du pavillon de thé Mittan dans le temple Ryōkō-in) », dans Gasetsu 画説, Tōkyō bijutsu kenkyūjō東京美術研究所, avril 1941.

11. Mori, Osamu, Katsura rikyū no kenkyū 桂離宮の研究 (Étude sur la villa Katsura), Tōkyō, Bunkasha 東都文化社, 1955 ; Katsura rikyū 桂離宮 (La Villa Katsura), Tōkyō, Shūeisha 集英社, 1967 ; Kobori Enshū no sakuji 小堀遠州の作事 (L’EEuvre de Kobori Enshū), Tōkyō, Kichikawa kōbunkan 吉川弘文館, Nara kokuritsu bunkazai kenkyūjo gakuhō jyūhachisatsu 奈良国立文化財 研究所学報第十八冊 $\left(18^{\mathrm{e}}\right.$ rapport du Centre de recherche national de Nara pour les propriétés culturelles), mars 1967.

12. Mori, Osamu, Kobori Enshū, op.cit., p. 272.

13. Mori, Osamu, Kobori Enshū no sakuji (L'CEuvre de Kobori Enshū), op.cit.

14. Kobori, Sôkei, 小堀 宗慶, Kobori Enshû no bi wo tazunete 小堀遠州の美を訪ねて (À la rencontre de l'esthétique de Kobori Ensh̄̄), Tōkyō, Shūeisha 集英社, 2010, 208 p. 
15. Shigemori, Mirei, , Kobori Enshū, op.cit., p. 181：「（前略）あくまでも人間としての自個の力 を信じた彼であり、人間の行為が、あらゆるものを制服することによって、自然も亦作り 得ることを信じた彼であった。だから、藝術の上に自然を生かすことは、自然を模倣した り、あるがま入の自然に從ふ必要はないと信じてるた。作者の創作力によって、別な角度 から、大きな自然を生み、叉は自然を發見することが出來ると云ふ自信をもってるた。こ の様な點を理解せぬ限り、遠州の藝術に對して理解し難い點があると言へるであらう。」 16. Mori, Osamu, Kobori Enshū, op.cit., p. 196：「遠州の庭園の意匠は自然順応にあった。それは 平安時代庭園の秘质書である『作庭記』に力説してあることであるが、遠州はまた平安時 代の庭園作家ったちの理想であった自然風景の利用も得意であった。」

\section{RÉSUMÉS}

À partir de l'analyse de deux biographies de Kobori Enshū, fameux paysagiste du début du $\mathrm{XVII}^{\mathrm{e}}$ siècle, nous nous proposons de réfléchir sur l'écriture de l'histoire des jardins japonais au $\mathrm{xx}^{\mathrm{e}}$ siècle. À première vue, tout semble opposer les deux principaux historiens des jardins japonais d'après-guerre : Shigemori Mirei et Mori Osamu. Le premier était un autodidacte, un artiste visionnaire et inspiré alors que le second était un universitaire qui a basé son travail sur les manuscrits et les fouilles. Si leurs interprétations divergent à propos du travail de Kobori Enshū, nous verrons dans cet article que chacun s'approprie et revisite à sa manière la vie et l'œuvre de ce personnage hors du commun. Et, détail intéressant, cette différence semble se retrouver dans leur travail en tant que paysagiste. La lecture de l'histoire des jardins japonais est donc primordiale pour comprendre l'évolution des jardins aujourd'hui.

Based on the analysis of two biographies of Kobori Enshū, famous landscaper from the early $17^{\text {th }}$ century, one offers to think over the writing of the history of Japanese gardens of the $20^{\text {th }}$ century. At first sight, everything seems to oppose the two main historians of the post-war Japanese gardens : Shigemori Mirei and Mori Osamu. The former was an autodidact, a visionary and an inspired artist, while the latter was a scholar who based his works on manuscripts, excavations and research. If their interpretations differ on Kobori Enshū's works, one shall see in this article that both of them take over the life and work of this exceptional character in order to revisit them in his own personal way. An interesting detail : this difference seems to be found in their works as landscapers. Thus, the reading of the history of Japanese gardens is paramount in order to understand the evolution of gardens nowadays.

\section{INDEX}

Mots-clés : Kobori Enshū, Shigemori Mirei, Mori Osamu, histoire, jardins japonais Keywords : Kobori Enshū, Shigemori Mirei, Mori Osamu, history, Japanese gardens 


\section{AUTEUR}

\section{EMMANUEL MARÈS}

Postdoctorant au CRCAO, chercheur invité à l'Institut national de recherche de Nara pour les propriétés culturelles importantes, et chercheur en histoire des jardins japonais. Membre du réseau Japarchi.

mamanum[at]gmail[dot]com 